\title{
UNIQUENESS OF RENORMALIZED SOLUTIONS \\ TO NONLINEAR ELLIPTIC EQUATIONS \\ WITH A LOWER ORDER TERM \\ AND RIGHT-HAND SIDE IN $L^{1}(\Omega)$
}

\author{
M.F. Betta ${ }^{1}$, A. Mercaldo ${ }^{2}$, F. Murat $^{3}$ And M.M. Porzio ${ }^{4}$
}

Abstract. In this paper we prove uniqueness results for the renormalized solution, if it exists, of a class of non coercive nonlinear problems whose prototype is

$$
\begin{cases}-\operatorname{div}\left(a(x)\left(1+|\nabla u|^{2}\right)^{\frac{p-2}{2}} \nabla u\right)+b(x)\left(1+|\nabla u|^{2}\right)^{\frac{\lambda}{2}}=f & \text { in } \Omega, \\ u=0 & \text { on } \partial \Omega\end{cases}
$$

where $\Omega$ is a bounded open subset of $\mathbb{R}^{N}, N \geq 2,2-1 / N<p<N, a$ belongs to $L^{\infty}(\Omega), a(x) \geq \alpha_{0}>0$, $f$ is a function in $L^{1}(\Omega), b$ is a function in $L^{r}(\Omega)$ and $0 \leq \lambda<\lambda^{*}(N, p, r)$, for some $r$ and $\lambda^{*}(N, p, r)$.

Mathematics Subject Classification. 35J25, 35J60.

Received February 11, 2002.

Dedicated to the memory of Jacques-Louis Lions, an exceptional mathematician and an exceptional man

\section{INTRODUCTION}

In the present paper, announced in [2], we prove uniqueness results for renormalized solutions of a class of problems whose prototype is

$$
\begin{cases}-\operatorname{div}\left(a(x)\left(1+|\nabla u|^{2}\right)^{\frac{p-2}{2}} \nabla u\right)+b(x)\left(1+|\nabla u|^{2}\right)^{\frac{\lambda}{2}}=f-\operatorname{div}(g) & \text { in } \Omega \\ u=0 & \text { on } \partial \Omega\end{cases}
$$

\footnotetext{
Keywords and phrases: Uniqueness, nonlinear elliptic equations, noncoercive problems, data in $L^{1}$.

1 Dipartimento di Matematica, Seconda Università di Napoli, via Vivaldi 43, 81100 Caserta, Italy; e-mail: mariafrancesca.betta@unina2.it

2 Dipartimento di Matematica e Applicazioni "R. Caccioppoli", Università degli Studi di Napoli "Federico II", Complesso Monte S. Angelo, via Cintia, 80126 Napoli, Italy; e-mail: mercaldo@unina.it

${ }^{3}$ Laboratoire Jacques-Louis Lions, Université Paris VI, Boîte courrier 187, 75252 Paris Cedex 05, France; e-mail: murat@ann.jussieu.fr

4 Facoltà di Scienze Matematiche, Fisiche e Naturali, Università degli Studi del Sannio, via Port'Arsa 11, 82100 Benevento, Italy; e-mail: porzio@unisannio.it
} 
where $\Omega$ is a bounded open subset of $\mathbb{R}^{N}, N \geq 2,2-1 / N<p<N$, a is a function belonging to $L^{\infty}(\Omega)$ such that $a(x) \geq \alpha_{0}>0, f$ belongs to $L^{1}(\Omega)$ and $g$ to $\left(L^{p^{\prime}}(\Omega)\right)^{N}, b$ belongs to some Lebesgue space $L^{r}(\Omega)$ and $0 \leq \lambda<\lambda^{*}(N, p, r)$ for some $\lambda^{*}(N, p, r)$ which is specified in Theorems 1.7, 1.11 and 1.16 below. We also prove comparison results.

We have proved in [3] an existence result for this type of problems when $1<p<N$ and $0 \leq \lambda \leq p-1$.

Problem (0.1) presents two difficulties: the first one is due to the term $f \in L^{1}(\Omega)$ in the right-hand side, which leads one to consider renormalized solutions; the second one is due to the term $b(x)\left(1+|\nabla u|^{2}\right)^{\frac{\lambda}{2}}$, which produces, in some sense, some non coerciveness in the operator.

Note that we confine ourselves to the case of an elliptic operator in divergence form with $L^{\infty}(\Omega)$ coefficients, which implies that the natural space (at least when $f=0$ and $0 \leq \lambda \leq p-1$ ) is the Sobolev space $W_{0}^{1, p}(\Omega)$. Note also that the problem under consideration is strongly related to maximum principle properties (or more exactly comparison properties) for solutions which belong to this type of Sobolev spaces. When smooth solutions are considered, there are a lot of uniqueness results, for $C^{2, \alpha}$ or $W^{2, p}$ solutions, but we will not try to give even a selected bibliography of them. On the other hand, there are few results in the framework we consider here.

When $b=0$ and $g=0$, the difficulty of the problem comes from the fact that the right-hand side belongs to $L^{1}(\Omega)$.

In the linear case (where $p=2$ ), Stampacchia defined in [18] a notion of solution of (0.1) by duality, for which he proved existence and uniqueness; he proved in particular that this solution belongs to $W_{0}^{1, q}(\Omega)$ for every $q<\frac{N}{N-1}$ and satisfies equation (0.1) in the distributional sense. Stampacchia's duality arguments have been extended to the nonlinear case when $p=2$ (see [15]), but not to the case $p \neq 2$.

In the general case where $p \neq 2$, three equivalent notions of solutions for problems of type $(0.1)$ with $b=0$ have been introduced: the notion of entropy solution, in $[1,5]$, the notion of SOLA (solution obtained as limit of approximations) in [7], and the notion of renormalized solution in [13-15]. Those three notions turn to be equivalent in the case where the right-hand side belongs to $L^{1}(\Omega)$ or to $L^{1}(\Omega)+W^{-1, p^{\prime}}(\Omega)$. In the above mentioned papers the authors proved the existence and uniqueness of such solutions (see also [12] for recent uniqueness results in the case where the right-hand side is a measure). Note that usual weak solutions are not well suited for this type of problems, since the solution does not, in general, belong to $W_{0}^{1, p}(\Omega)$ when $f \in L^{1}(\Omega)$, but only to the space $W_{0}^{1, q}(\Omega)$ for every $q<\frac{N(p-1)}{N-1}$, and since a classical counterexample ([17], see also [16]) shows that, in the linear case, such a solution is not unique.

Let us now pass to the case where $f=0$ (and therefore where the right-hand side belongs to $W^{-1, p^{\prime}}(\Omega)$, so that usual weak solutions are well suited for the problem) but where $b \neq 0$. In such a setting the only uniqueness result we know is the result of Bottaro and Marina [6], which states that in the linear case (where $p=2$ and $\lambda=1)$, there is existence and uniqueness of a solution of $(0.1)$ for every $b \in L^{N}(\Omega)$, even with $\|b\|_{L^{N}(\Omega)}$ very large (it is easy to see that the linear operator defined by $(0.1)$ is coercive when $\|b\|_{L^{N}(\Omega)}$ is small, and in this case existence and uniqueness are immediate consequences of Lax-Milgram lemma).

In the present paper we face both difficulties $\left(f \in L^{1}(\Omega)\right.$ and $b \in L^{r}(\Omega)$ with $\|b\|_{L^{r}(\Omega)}$ large). We prove uniqueness for some $r$ and for $\lambda$ satisfying

$$
0 \leq \lambda<\lambda^{*}(N, p, r)
$$

where $\lambda^{*}(N, p, r)$ is a complicated expression of $N, p$ and $r$, which in general does not coincide with $p-1$.

Since in [3] we proved the existence of renormalized solutions for problems of type (0.1) with $b$ in the Lorentz space $b \in L^{N, 1}(\Omega)$ and $0 \leq \lambda \leq p-1$, the uniqueness results of the present paper are both surprising and unsatisfactory: in some cases, we have proved existence and uniqueness, but in other cases, existence but not uniqueness, and finally in some other ones, uniqueness but not existence. Let us however emphasise that these difficulties are not primarily related to the fact that we are dealing with a right-hand side in $L^{1}(\Omega)$ 
and renormalized solutions, but that the same difficulties appear in the case where one deals with usual weak solutions for a right-hand side in $W^{-1, p^{\prime}}(\Omega)$ : we will prove uniqueness results in this more classical framework in $[4]$.

The difference between the restrictions on $\lambda$ concerning existence and uniqueness results is mainly due to the fact that we work in a framework with $p$-coerciveness as far as existence is involved, while for uniqueness we are dealing with weighted quadratic coerciveness. Let us explain this in the simple case where $u_{1}-u_{2}$ can be used as test function in the difference of the equations satisfied by $u_{1}$ and $u_{2}$. This provides a formal estimate of the type

$$
\begin{aligned}
C \int_{\Omega}\left(1+\left|\nabla u_{1}\right|+\left|\nabla u_{2}\right|\right)^{p-2}\left|\nabla u_{1}-\nabla u_{2}\right|^{2} & \leq \int_{\Omega} a(x)\left[\left(1+\left|\nabla u_{1}\right|^{2}\right)^{\frac{p-2}{2}} \nabla u_{1}-\left(1+\left|\nabla u_{2}\right|^{2}\right)^{\frac{p-2}{2}} \nabla u_{2}\right]\left(\nabla u_{1}-\nabla u_{2}\right) \\
& \leq \int_{\Omega} b(x)\left|\left(1+\left|\nabla u_{1}\right|^{2}\right)^{\frac{\lambda}{2}}-\left(1+\left|\nabla u_{2}\right|^{2}\right)^{\frac{\lambda}{2}}\right|\left|u_{1}-u_{2}\right| \\
& \leq C \int_{\Omega} b(x)\left(1+\left|\nabla u_{1}\right|+\left|\nabla u_{2}\right|\right)^{\lambda-1}\left|\nabla u_{1}-\nabla u_{2}\right|\left|u_{1}-u_{2}\right| .
\end{aligned}
$$

We then use Hölder inequality to make the weighted norm

$$
\int_{\Omega}\left(1+\left|\nabla u_{1}\right|+\left|\nabla u_{2}\right|\right)^{p-2}\left|\nabla u_{1}-\nabla u_{2}\right|^{2}
$$

appear in the right-hand side, which explains the various cases which appear according to the values of $p$. This leads to computations which are sometimes technical and are not completely satisfactory.

The previous computation also explains why we considered a non degenerated operator $-\operatorname{div}(a(x)(1+$ $\left.\left.|\nabla u|^{2}\right)^{\frac{p-2}{2}} \nabla u\right)$ in place of the (more classical) $p$-Laplace operator $-\operatorname{div}\left(|\nabla u|^{p-2} \nabla u\right)$. Indeed the weight $\left(1+\left|\nabla u_{1}\right|+\left|\nabla u_{2}\right|\right)^{\lambda-1}$ naturally appears in the right-hand side of the previous estimate, and this leads to the operator considered here. However in the case where $p<2$, the weight $\left(1+\left|\nabla u_{1}\right|+\left|\nabla u_{2}\right|\right)^{p-2}$ can be replaced by $\left(\left|\nabla u_{1}\right|+\left|\nabla u_{2}\right|\right)^{p-2}$, which explains why we can consider the usual $p$-Laplace operator (which is not degenerated in 0$)$ in this case.

Let us finally say a few words about the two Appendices of the present paper. In the first one, we establish a new property concerning the difference of two renormalized solutions when $p<2$ : while it was known ([8], Theorem 9.1) that, when $p>2$, the difference of two renormalized solutions satisfies $T_{k}\left(u_{1}-u_{2}\right) \in W_{0}^{1, p}(\Omega)$ for every $k$, no similar result was known for the case $p<2$. When $\frac{3 N-2}{2 N-1}<p \leq 2$, we prove here that the difference of two renormalized solutions satisfies $T_{k}\left(u_{1}-u_{2}\right) \in W_{0}^{1, s}(\Omega)$ for $s<\frac{2 N(p-1)}{N-(2-p)}$.

In the second Appendix we revisit the result of [1] which asserts that

$$
\int_{\Omega}\left|\nabla T_{k}(u)\right|^{p} \leq M k, \quad \forall k>0,
$$

implies

$$
\left\||\nabla u|^{p-1}\right\|_{L^{N^{\prime}, \infty}(\Omega)} \leq C_{0} M
$$

We generalize this result in two different ways: first to the case where in $(0.2) M k$ is replaced by $M k^{\theta}$ with $0<\theta<p$, and second to the case where $(0.2)$ is replaced by

$$
\int_{\Omega} v(x)\left|\nabla T_{k}(u)\right|^{p} \leq M k, \quad \forall k>0,
$$

with a weight $v(x) \geq A_{0}>0$. 


\section{Definitions And main Results}

In this section, we recall the definition of renormalized solution for nonlinear elliptic problems with right-hand side in $L^{1}(\Omega)+W^{-1, p^{\prime}}(\Omega)(c f .[1,5,7,8,13-15])$, and we state our uniqueness results.

\subsection{Assumptions and definition of a renormalized solution}

For $1<r<\infty$, the Lorentz space $L^{r, \infty}(\Omega)$ is the space of Lebesgue measurable functions such that

$$
\|f\|_{L^{r, \infty}(\Omega)}=\sup _{t>0} t[\operatorname{meas}\{x \in \Omega:|f(x)|>t\}]^{1 / r}<+\infty,
$$

endowed with the norm defined by (1.1). Recall that for every $1<s<r<\infty$, one has

$$
L^{r}(\Omega) \subset L^{r, \infty}(\Omega) \subset L^{s}(\Omega) .
$$

For $k>0$, denote by $T_{k}: \mathbb{R} \rightarrow \mathbb{R}$ the usual truncation at level $k$, that is

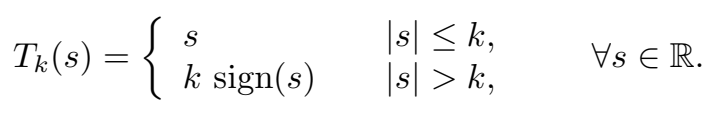

Consider a measurable function $u$ defined almost everywhere on $\Omega$ which is finite almost everywhere and satisfies $T_{k}(u) \in W_{0}^{1, p}(\Omega)$ for every $k>0$. Then there exists (see e.g. [1], Lemma 2.1) an unique measurable vector function $v$ defined almost everywhere on $\Omega$ such that

$$
\nabla T_{k}(u)=v \chi_{\{|u| \leq k\}} \quad \text { almost everywhere in } \quad \Omega, \quad \forall k>0 .
$$

We define the gradient $\nabla u$ of $u$ as this function $v$, and denote $\nabla u=v$. Note that the previous definition does not coincide with the definition of the distributional gradient. However if $v \in\left(L_{\mathrm{loc}}^{1}(\Omega)\right)^{N}$, then $u \in W_{\mathrm{loc}}^{1,1}(\Omega)$ and $v$ is the distributional gradient of $u$. In contrast there are examples of functions $u \notin L_{\text {loc }}^{1}(\Omega)$ (and thus for which the gradient in the distributional sense is not defined) for which there exists the gradient $\nabla u$ defined in the previous sense (see Remarks 2.10 and 2.11, Lemma 2.12 and Example 2.16 in [8]).

In the present paper we consider a nonlinear elliptic problem which can formally be written as

$$
\left\{\begin{array}{lll}
-\operatorname{div}(a(x, \nabla u))+H(x, \nabla u)+G(x, u)=f-\operatorname{div}(g) & \text { in } \quad \Omega \\
u=0 & \text { on } \quad \partial \Omega .
\end{array}\right.
$$

Here $\Omega$ is a bounded open subset of $\mathbb{R}^{N}, N \geq 2, p$ is a real number with $1<p<N$, and $a: \Omega \times \mathbb{R}^{N} \rightarrow \mathbb{R}^{N}$ is a Carathéodory function satisfying

$$
\begin{gathered}
a(x, \xi) \xi \geq \alpha|\xi|^{p}, \quad \alpha>0, \\
|a(x, \xi)| \leq c\left[|\xi|^{p-1}+a_{0}(x)\right], \quad a_{0}(x) \in L^{p^{\prime}}(\Omega), \quad c>0,
\end{gathered}
$$

for almost every $x \in \Omega$ and for every $\xi \in \mathbb{R}^{N}$. 
We assume that $a(x, \xi)$ is strongly monotone ${ }^{5}$, i.e.

$$
\left\{\begin{array}{l}
(a(x, \xi)-a(x, \eta), \xi-\eta) \geq \beta(A(x)+|\xi|+|\eta|)^{p-2}|\xi-\eta|^{2} \\
\beta>0, \quad A^{p-1} \in L^{N^{\prime}, \infty}(\Omega), \quad A(x) \geq 0
\end{array}\right.
$$

for almost every $x \in \Omega$ and for every $\xi \in \mathbb{R}^{N}, \eta \in \mathbb{R}^{N}$.

Moreover $H: \Omega \times \mathbb{R}^{N} \rightarrow \mathbb{R}$ is a Carathéodory function with

$$
H(x, 0) \in L^{1}(\Omega)
$$

such that $H(x, \xi)$ is locally Lipschitz continuous with respect to $\xi$, i.e.

$$
\left\{\begin{array}{l}
|H(x, \xi)-H(x, \eta)| \leq b(x)\left(A_{0}+|\xi|+|\eta|\right)^{\sigma_{A_{0}}}(B(x)+|\xi|+|\eta|)^{\sigma_{B}}|\xi-\eta|, \\
b \in L^{r}(\Omega), b(x) \geq 0, \sigma_{A_{0}} \geq 0, \sigma_{B} \geq 0, A_{0} \in \mathbb{R}, A_{0} \geq 0, B^{p-1} \in L^{N^{\prime}, \infty}(\Omega), B(x) \geq 0,
\end{array}\right.
$$

for almost every $x \in \Omega$ and for every $\xi \in \mathbb{R}^{N}, \eta \in \mathbb{R}^{N}$, where $\sigma_{A_{0}}, \sigma_{B}$ and $r$ are constants to be specified in the statements of Theorems 1.7, 1.11, 1.16 below.

We also assume that $G: \Omega \times \mathbb{R} \rightarrow \mathbb{R}$ is a Carathéodory function such that

$$
(G(x, s)-G(x, t))(s-t) \geq 0
$$

for almost every $x \in \Omega$ and for every $s \in \mathbb{R}, t \in \mathbb{R}$.

Finally we assume that

$$
f \in L^{1}(\Omega), \quad g \in\left(L^{p^{\prime}}(\Omega)\right)^{N}
$$

Remark 1.1. The model case for $a(x, \xi)$ satisfying assumptions $(1.5)-(1.7)$ is

$$
\left\{\begin{array}{l}
a(x, \xi)=\gamma(x)\left(\Gamma(x)^{2}+|\xi|^{2}\right)^{\frac{p-2}{2}} \xi \\
\gamma \in L^{\infty}(\Omega), \quad \gamma(x) \geq \gamma_{0}>0, \quad \Gamma \in L^{\infty}(\Omega), \quad \Gamma(x) \geq \Gamma_{0}
\end{array}\right.
$$

in which we will assume $\Gamma_{0} \geq 0$ when $p \leq 2$, and $\Gamma_{0}>0$ when $p>2$. Similarly, the model case for $H(x, \xi)$ is

$$
\left\{\begin{array}{l}
H(x, \xi)=b(x)\left(\hat{A}(x)^{2}+|\xi|^{2}\right)^{\lambda / 2}\left(B(x)^{2}+A_{0}^{2}+|\xi|^{2}\right)^{\mu / 2}, \\
b \in L^{r}(\Omega), \quad b(x) \geq 0, \quad \hat{A} \in L^{\infty}(\Omega), \quad A_{0} \geq \hat{A}(x) \geq 0, \quad B^{p-1} \in L^{N^{\prime}, \infty}(\Omega), \quad B(x) \geq 0
\end{array}\right.
$$

for which one easily proves that

$$
\left|\frac{\partial H}{\partial \xi}(x, \xi)\right| \leq C b(x)(\hat{A}(x)+|\xi|)^{\lambda-1}\left(B(x)+A_{0}+|\xi|\right)^{\mu} .
$$

Therefore taking $\sigma_{A_{0}}=\lambda-1$ and $\sigma_{B}=\mu, H$ defined by (1.13) satisfies (1.9) when $\lambda \geq 1$ and $\mu \geq 0$.

\footnotetext{
${ }^{5}$ When $p<2$ and $A(x)=0$, the term $\frac{|\xi-\eta|^{2}}{(A(x)+|\xi|+|\eta|)^{2-p}}$ is not defined for $\xi=\eta=0$. We make the convention that this quantity is 0 in that case.
} 
Remark 1.2. Observe that two different functions $A_{0}$ and $B(x)$ appear in the right-hand side of hypothesis (1.9). Of course replacing $A_{0}$ and $B(x)$ by $A_{0}+B(x)$, one can assume hypothesis (1.9) with only one function in the right-hand side, namely hypothesis (1.9) with $b(x)\left(A_{0}+B(x)+|\xi|+|\eta|\right)^{\sigma_{A_{0}}+\sigma_{B}}|\xi-\eta|$ as righthand side, but hypothesis (1.9) is a little bit more general and will be used in this form in the proof.

Remark 1.3. In the whole of the present paper we assume that $N \geq 2$ and $1<p<N$. The first assumption, $N \geq 2$, is dictated by the second one, $1<p<N$. The latest follows from the fact that we consider here the case where the right-hand side is in $L^{1}(\Omega)$ : when $p>N$, one has $L^{1}(\Omega) \subset W^{-1, p^{\prime}}(\Omega)$, which implies that the right-hand side belongs to the dual space $W^{-1, p^{\prime}}(\Omega)$. This case will be considered in [4], and thus is excluded in the present paper. Actually we also exclude the case $p=N$ because in that case the Sobolev embedding does not hold and has to be replaced by the Trudinger-Moser embedding, which leads to very technical problems. When $p=N$, existence and uniqueness results of a solution in the distributional sense have been proved in [9-11] in the case where $H=0$.

Definition 1.4. We say that $u$ is a renormalized solution of (1.4) if it satisfies the following conditions:

$u$ is measurable on $\Omega, \quad$ almost everywhere finite, and such that $T_{k}(u) \in W_{0}^{1, p}(\Omega), \quad \forall k>0$;

$$
|u|^{p-1} \in L^{\frac{N}{N-p}, \infty}(\Omega)
$$

the gradient $\nabla u$ introduced in (1.3) satisfies:

$$
\begin{aligned}
& |\nabla u|^{p-1} \quad \text { belongs to } L^{N^{\prime}, \infty}(\Omega), \\
& \lim _{n \rightarrow+\infty} \frac{1}{n} \int_{n \leq|u|<2 n} a(x, \nabla u) \cdot \nabla u=0
\end{aligned}
$$

and finally

$$
\begin{aligned}
\int_{\Omega} a(x, \nabla u) \cdot \nabla u h^{\prime}(u) v+\int_{\Omega} a(x, \nabla u) \cdot \nabla v h(u) \\
\quad+\int_{\Omega} H(x, \nabla u) h(u) v+\int_{\Omega} G(x, u) h(u) v \\
=\int_{\Omega} f h(u) v+\int_{\Omega} g \cdot \nabla u h^{\prime}(u) v+\int_{\Omega} g \cdot \nabla v h(u),
\end{aligned}
$$

for every $v \in W^{1, p}(\Omega) \cap L^{\infty}(\Omega)$, for all $h \in W^{1, \infty}(\mathbb{R})$ with compact support in $\mathbb{R}$, which are such that $h(u) v \in$ $W_{0}^{1, p}(\Omega)$.

Since $h(u) v \in W_{0}^{1, p}(\Omega)$ and since $\operatorname{supp}(h) \subset[-2 n, 2 n]$ (for a suitable $n>0$ depending on $h$ ), we can rewrite (1.18) as follows

$$
\begin{aligned}
\int_{\Omega} a( & \left.x, \nabla T_{2 n}(u)\right) \cdot \nabla T_{2 n}(u) h^{\prime}(u) v+\int_{\Omega} a\left(x, \nabla T_{2 n}(u)\right) \cdot \nabla v h(u) \\
& +\int_{\Omega} H\left(x, \nabla T_{2 n}(u)\right) h(u) v+\int_{\Omega} G\left(x, T_{2 n}(u)\right) h(u) v \\
= & \int_{\Omega} f h(u) v+\int_{\Omega} g \cdot \nabla T_{2 n}(u) h^{\prime}(u) v+\int_{\Omega} g \cdot \nabla v h(u) .
\end{aligned}
$$


Let us observe that every integral in (1.19) is well defined in view of (1.5)-(1.11) since $T_{2 n}(u) \in W_{0}^{1, p}(\Omega)$.

Remark 1.5. As already said in the introduction, the notion of renormalized solution was introduced in [13-15] when $H=G=0$. This notion is equivalent to the notion of entropy solution introduced in $[1,5]$ and to the notion of SOLA introduced in [7]. In the case where $H=G=0$, it is proved in these papers that there exists such a solution, which is unique.

The definition given above is a natural extension to the case $H \neq 0, G \neq 0$ of this three equivalent definitions. The goal of the present paper is to prove the uniqueness of such a solution for $H \neq 0$ satisfying some local Lipschitz continuity condition (see (1.9)). Recall that we proved existence of such a solution in [3] when $H$ satisfies the growth condition

$$
\left\{\begin{array}{l}
|H(x, \xi)| \leq b_{0}(x)|\xi|^{p-1}+b_{1}(x) \\
b_{0} \in L^{N, 1}(\Omega), \quad b_{1} \in L^{1}(\Omega)
\end{array}\right.
$$

Remark 1.6. If $u$ is a renormalized solution of (1.4), if $\frac{1}{r}+\frac{\sigma_{A_{0}}+\sigma_{B}+1}{N^{\prime}}<1$ and if $G(x, u)$ belongs to $L^{1}(\Omega)$, then $u$ is also a distributional solution in the sense that $u$ satisfies

$$
\int_{\Omega} a(x, \nabla u) \cdot \nabla \phi+\int_{\Omega} H(x, \nabla u) \phi+\int_{\Omega} G(x, u) \phi=\int_{\Omega} f \phi+\int_{\Omega} g \cdot \nabla \phi,
$$

for all $\phi \in C_{0}^{\infty}(\Omega)$.

Indeed if $u$ is a renormalized solution of (1.4), we know that $u$ is measurable and almost everywhere finite in $\Omega$, and that $T_{k}(u) \in W_{0}^{1, p}(\Omega)$ for every $k>0$, which allows one to define $\nabla u$ in the sense of (1.3). We also know that $|\nabla u|^{p-1}$ then belongs to $L^{N^{\prime}, \infty}(\Omega)$ so that $|a(x, \nabla u)|$ belongs to $L^{N^{\prime}, \infty}(\Omega)$ by the growth condition (1.6). Moreover $H(x, \nabla u)$ belongs to $L^{1}(\Omega)$ in view of (1.8), (1.9) and of $\frac{1}{r}+\frac{\sigma_{A_{0}}+\sigma_{B}+1}{N^{\prime}}<1$. Taking $\phi \in C_{0}^{\infty}(\Omega)$ and $h_{n}$ defined by

$$
h_{n}(s)= \begin{cases}0 & |s|>2 n \\ \frac{2 n-|s|}{n} & n<|s| \leq 2 n \\ 1 & |s| \leq n\end{cases}
$$

and letting $n$ tend to infinity, we obtain (1.21).

Observe also that when $p>2-\frac{1}{N}$, one has $N^{\prime}(p-1)>1$; in this case, the gradient $\nabla u$, defined by (1.3), satisfies (1.16), and then $u$ belongs to $W_{0}^{1, q}(\Omega)$, for every $q<\frac{N(p-1)}{N-1}$, while $\nabla u$ is now the distributional gradient of $u$ (see [8], Remark 2.10). In such a case the renormalized solution $u$ belongs to the Sobolev space $W_{0}^{1, q}(\Omega)$, and satisfies (1.21), i.e. (1.4) in the sense of distributions. Nevertheless we have to use the notion of renormalized solution, since it is well known that even in the linear case where $p=2$ and $H=0$, the solution $u$ of

$$
\begin{cases}u \in W_{0}^{1, q}(\Omega), \quad \forall q<\frac{N}{N-1}, & \\ -\operatorname{div}(A(x) \nabla u)=f \quad \text { in } \quad \mathcal{D}^{\prime}(\Omega),\end{cases}
$$

is in general not unique ( $c f .[17]$, see also [16]).

\subsection{Uniqueness results}

Under the assumptions stated above, we will prove three uniqueness results (Theorems $1.7,1.11,1.16$ ) for some values of $\sigma_{A_{0}}$ and $\sigma_{B}$, according to the values of $p\left(2-\frac{1}{N}<p \leq 2,2<p<\frac{2 N-4}{N-3}\right.$, and $\left.\frac{2 N-4}{N-3} \leq p<N\right)$. 
These uniqueness results correspond to proofs which use different techniques. We did our best to optimise the various parameters which enter in these proofs, and we hope that we obtained the best possible results, i.e. the largest sets of parameters $\sigma_{A_{0}}$ and $\sigma_{B}$ for which uniqueness holds.

Observe that we do not have any uniqueness result when $1<p \leq 2-\frac{1}{N}$. However this restriction on the values of $p$ is not related to the (same) restriction $p>2-\frac{1}{N}$ which appears as a sufficient condition for the renormalized solution of (1.4) to belong to some Sobolev space (see Remark 1.6 above).

Theorem 1.7. Let $N \geq 2$ and $p$ be such that

$$
\begin{cases}2-\frac{1}{N}<p<2, & \text { if } \quad N=2 \\ 2-\frac{1}{N}<p \leq 2, & \text { if } \quad N \geq 3\end{cases}
$$

We assume that (1.5)-(1.11) are satisfied with

$$
\begin{gathered}
A(x) \geq A_{0} \geq 0 \\
\frac{N(p-1)}{1-N(2-p)}<r \leq+\infty, \\
0 \leq \sigma_{A_{0}}<\sigma^{*}(N, p, r), \\
0 \leq \sigma_{B}<\sigma^{*}(N, p, r)-\sigma_{A_{0}},
\end{gathered}
$$

where

$$
\sigma^{*}(N, p, r)=\frac{1-N(2-p)}{N-1}-\frac{N(p-1)}{N-1} \frac{1}{r} .
$$

Let $u_{1}$ and $u_{2}$ be two renormalized solutions of (1.4) such that

$$
G\left(x, u_{1}\right) \in L^{1}(\Omega), \quad G\left(x, u_{2}\right) \in L^{1}(\Omega) .
$$

Then $u_{1}=u_{2}$.

Remark 1.8. Under the assumptions of Theorem 1.7, we also have the following comparison result: if $u_{1}$ and $u_{2}$ are two renormalized solutions of (1.4), which correspond to two functions $f_{1}$ and $f_{2}$ (with the same $g$ ) such that

$$
f_{1} \leq f_{2} \text { a.e. in } \Omega
$$

then

$$
u_{1} \leq u_{2} \text { a.e. in } \Omega \text {. }
$$

Remark 1.9. Since we assumed $1<p<N$ (see Remark 1.3 above), we have to restrict to $\frac{3}{2}<p<2$ in the case where $N=2$, while $p=2$ is possible when $N \geq 3$.

Observe that when $2-\frac{1}{N}<p$ (which is equivalent to $\frac{1-N(2-p)}{N-1}>0$ ) and when $r$ satisfies (1.26), then $\sigma^{*}(N, p, r)$ defined by (1.29) satisfies $\sigma^{*}(N, p, r)>0$. Figure 1 shows the set (a triangle) of the values $\left(\sigma_{A_{0}}, \sigma_{B}\right)$ for which hypotheses of Theorem 1.7 holds true. This set is not empty. 


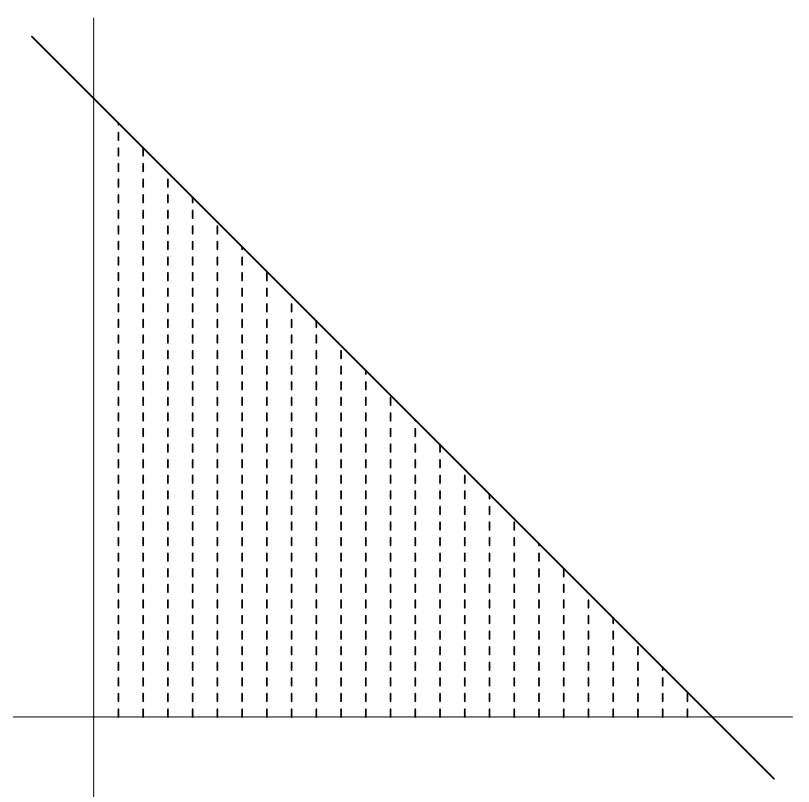

Figure 1. Values of $\left(\sigma_{A_{0}}, \sigma_{B}\right)$ for which Theorem 1.7 holds true. The horizontal axis gives the values of $\sigma_{A_{0}}$ and the vertical axis the values of $\sigma_{B}$. Theorem 1.7 holds true when the values of $\left(\sigma_{A_{0}}, \sigma_{B}\right)$ are inside the triangle defined by the vertical axis $\sigma_{A_{0}}=0$, the horizontal axis $\sigma_{B}=0$, and the oblique line $\sigma_{A_{0}}+\sigma_{B}=\sigma^{*}$.

Remark 1.10. Let us compare the hypotheses of Theorem 1.7 with those of the existence theorem we proved in [3]. For that we consider the model case where

$$
H(x, \xi)=\left(A_{0}+|\xi|^{2}\right)^{\lambda / 2}, \quad A_{0} \geq 0, \quad \lambda \geq 0
$$

It is easy to see that in this case, equation (1.9) holds with $b=$ constant, $r=\infty, A_{0}>0$ (and also $A_{0}=0$ if $\lambda \geq 1), \sigma_{A_{0}}=(\lambda-1)^{+}=\max \{\lambda-1,0\}, B=1$ and $\sigma_{B}=0$. In this case, Theorem 1.7 implies the uniqueness of the renormalized solution when

$$
(\lambda-1)^{+}<\sigma^{*}(N, p, \infty)=\frac{1-N(2-p)}{(N-1)},
$$

i.e.

$$
0 \leq \lambda<\frac{N(p-1)}{N-1}
$$

On the other hand, we proved in [3] that there exists a renormalized solution when

$$
0 \leq \lambda \leq p-1
$$

Therefore we have proved uniqueness for $\lambda$ in the interval where we proved existence, but also in the interval $p-1<\lambda<\frac{N(p-1)}{N-1}$, in which case existence is not proved. 
Theorem 1.11. Let $N \geq 3$ and $p$ be such that

$$
\begin{cases}2<p<3, & \text { if } \quad N=3 \\ 2<p<\frac{2 N-4}{N-3}, & \text { if } \quad N \geq 4\end{cases}
$$

We assume that (1.5)-(1.11) are satisfied with

$$
\begin{gathered}
A(x) \geq A_{0}>0, \\
\frac{2 N(p-1)(N+p-2)}{(N+p-2)[N(p-2)+p]-p(p-2)(N-1)^{2}}<r \leq+\infty, \\
0 \leq \sigma_{A_{0}}<\sigma^{*}(N, p, r), \\
0 \leq \sigma_{B}<\min \left\{\sigma^{*}(N, p, r)-\sigma_{A_{0}}, \sigma^{*}(N, p, r)-\rho^{*}(N, p)\right\},
\end{gathered}
$$

where

$$
\left\{\begin{array}{l}
\sigma^{*}(N, p, r)=\frac{N(p-2)+p}{2(N-1)}-\frac{N(p-1)}{N-1} \frac{1}{r} \\
\rho^{*}(N, p)=\frac{p(p-2)(N-1)}{2(N+p-2)}
\end{array}\right.
$$

Let $u_{1}$ and $u_{2}$ be two renormalized solutions of (1.4) such that

$$
G\left(x, u_{1}\right) \in L^{1}(\Omega), \quad G\left(x, u_{2}\right) \in L^{1}(\Omega) .
$$

Then $u_{1}=u_{2}$.

Remark 1.12. Under the assumptions of Theorem 1.11, we also have the following comparison result: if $u_{1}$ and $u_{2}$ are two renormalized solutions of (1.4), which correspond to two functions $f_{1}$ and $f_{2}$ (with the same $g$ ) such that

then

$$
f_{1} \leq f_{2} \text { a.e. in } \Omega
$$

$$
u_{1} \leq u_{2} \text { a.e. in } \Omega .
$$

Remark 1.13. Observe that $\frac{2 N-4}{N-3} \leq N$ when $N \geq 4$, so that (1.31) implies that $2<p<N$.

Observe also that $(N+p-2)[N(p-2)+p]-p(p-2)(N-1)^{2}>0$ when $p$ satisfies $(1.31)$, and that $r$ satisfies (1.33) if and only if

$$
\frac{N(p-2)+p}{2(N-1)}-\frac{N(p-1)}{N-1} \frac{1}{r}-\frac{p(p-2)(N-1)}{2(N+p-2)}>0 .
$$

Therefore, when (1.33) is satisfied, one has

$$
\rho^{*}(N, p)<\sigma^{*}(N, p, r)
$$

and Figure 2 shows the set of the values $\left(\sigma_{A_{0}}, \sigma_{B}\right)$ for which hypotheses of Theorem 1.11 hold true. This set is not empty. 


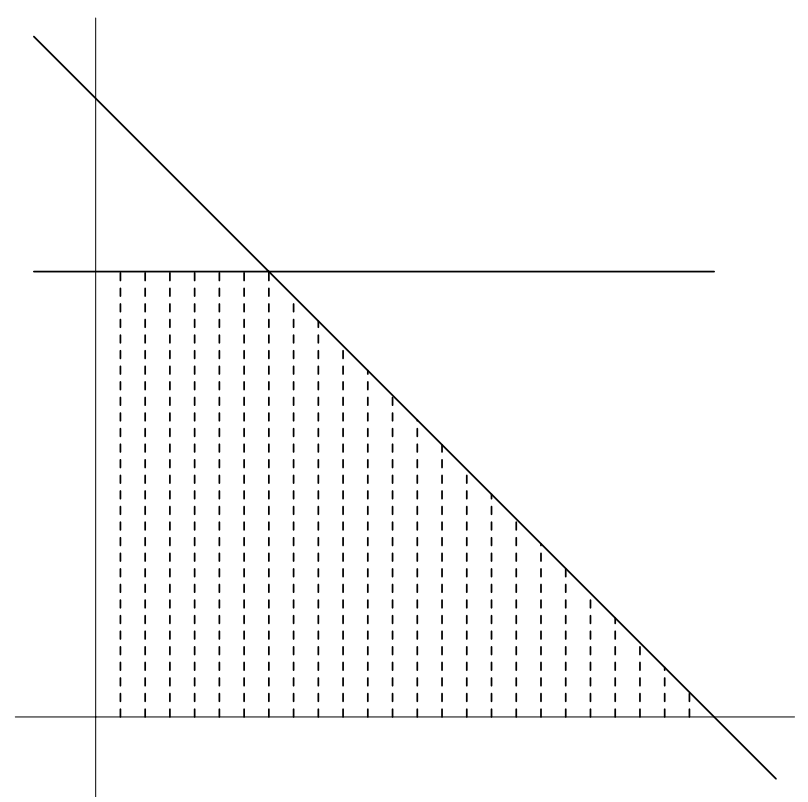

Figure 2. Values of $\left(\sigma_{A_{0}}, \sigma_{B}\right)$ for which Theorem 1.11 holds true. The horizontal axis gives the values of $\sigma_{A_{0}}$ and the vertical axis the values of $\sigma_{B}$. Theorem 1.11 holds true when the values of $\left(\sigma_{A_{0}}, \sigma_{B}\right)$ are inside the trapezium defined by the vertical axis $\sigma_{A_{0}}=0$, the horizontal axis $\sigma_{B}=0$, the horizontal line $\sigma_{B}=\sigma^{*}-\rho^{*}$, and the oblique line $\sigma_{A_{0}}+\sigma_{B}=\sigma^{*}$.

Remark 1.14. Let us compare the hypotheses of Theorem 1.11 with those of the existence theorem we proved in [3]. For that we consider the model case where

$$
H(x, \xi)=\left(1+|\xi|^{2}\right)^{\lambda / 2}, \quad \lambda \geq 0
$$

for which it is easy to see that (1.9) holds with $b=$ constant, $r=\infty, A_{0}=1, \sigma_{A_{0}}=(\lambda-1)^{+}, B=1$ and $\sigma_{B}=0$. In this case, Theorem 1.11 implies the uniqueness of the renormalized solution when

$$
(\lambda-1)^{+}<\sigma^{*}(N, p, \infty)=\frac{N(p-2)+p}{2(N-1)},
$$

i.e.

$$
0 \leq \lambda<\frac{(N+1) p-2}{2(N-1)}
$$

On the other hand, we proved in [3] that there exists a renormalized solution when

$$
0 \leq \lambda \leq p-1
$$

But when (1.31) holds, one has

$$
p-1<\frac{(N+1) p-2}{2(N-1)},
$$

and therefore we have proved uniqueness for $\lambda$ in the interval where we proved existence, but also in the interval $p-1<\lambda<\frac{(N+1) p-2}{2(N-1)}$, in which case existence is not proved. 
Remark 1.15. In this remark we consider the case where $N \geq 3$ and $p=2$, which is a borderline case between Theorems 1.7 and 1.11 .

In Theorem 1.7, $\sigma^{*}(N, p, r)$ is defined for $2-\frac{1}{N}<p \leq 2$, while in Theorem 1.11, $\sigma^{*}(N, p, r)$ and $\rho^{*}(N, p)$ are defined for $2<p<\frac{2 N-4}{N-3}$. But when $p>2$ tends to 2 , formula (1.36) show that

$$
\begin{gathered}
\sigma^{*}(N, p, r) \rightarrow \sigma^{*}(N, 2, r), \\
\rho^{*}(N, p) \rightarrow 0,
\end{gathered}
$$

which means that the results of Theorems 1.7 and 1.11 match when $p=2$.

Theorem 1.16. Let $N \geq 5$ and $p$ be such that

$$
\frac{2 N-4}{N-3} \leq p<N
$$

We assume that (1.5)-(1.11), are satisfied with

$$
\begin{gathered}
A(x) \geq A_{0}>0, \\
N<r \leq+\infty, \\
0 \leq \sigma_{A_{0}}<\sigma^{*}(N, p, r), \\
0 \leq \sigma_{B}<\sigma^{*}(N, p, r)-\sigma_{A_{0}}, \\
(N-1) \sigma_{B}\left[2(p-1)-\sigma_{B}\right]<\sigma_{A_{0}}\left[2(p-1)-(N-1) \sigma_{A_{0}}\right],
\end{gathered}
$$

where

$$
\sigma^{*}(N, p, r)=\frac{2(p-1)}{N-1}-\frac{2 N(p-1)}{(N-1)} \frac{1}{r} .
$$

Let $u_{1}$ and $u_{2}$ be two renormalized solutions of (1.4) such that

$$
G\left(x, u_{1}\right) \in L^{1}(\Omega), \quad G\left(x, u_{2}\right) \in L^{1}(\Omega) .
$$

Then $u_{1}=u_{2}$.

Remark 1.17. Under the assumptions of Theorem 1.16, we also have the following comparison result: if $u_{1}$ and $u_{2}$ are two renormalized solutions of (1.4), which correspond to two functions $f_{1}$ and $f_{2}$ (with the same $g$ ) such that

then

$$
f_{1} \leq f_{2} \text { a.e. in } \Omega,
$$$$
u_{1} \leq u_{2} \quad \text { a.e. in } \Omega \text {. }
$$ 
Remark 1.18. Observe that (1.39) implies that $N>4$.

Observe also that, when $r$ satisfies (1.41), i.e. $N<r \leq+\infty$, one has

$$
\sigma^{*}(N, p, r)=\frac{2(p-1)}{N-1}\left(1-\frac{N}{r}\right)>0
$$

On the other hand, inequality (1.44) is equivalent to

$$
\sigma_{B}^{2}-2(p-1) \sigma_{B}-\sigma_{A_{0}}^{2}+\frac{2(p-1)}{N-1} \sigma_{A_{0}}>0
$$

which means that the part of the boundary of the set of admissible values of $\left(\sigma_{A_{0}}, \sigma_{B}\right)$ defined by (1.44) is a branch of the hyperbola

$$
\mathcal{H}=\left\{\left(\sigma_{A_{0}}, \sigma_{B}\right):\left(\sigma_{B}-(p-1)\right)^{2}-\left(\sigma_{A_{0}}-\frac{p-1}{N-1}\right)^{2}=(p-1)^{2}-\left(\frac{p-1}{N-1}\right)^{2}\right\} .
$$

Note that $(0,0) \in \mathcal{H}$ and that

$$
\left\{\left(\sigma_{A_{0}}, \sigma_{B}\right) \in \mathcal{H}: 0 \leq \sigma_{A_{0}}<\sigma^{*}(N, p, r)\right\} \subset\left\{\sigma_{B} \geq 0\right\}
$$

Figure 3 shows the set of the values $\left(\sigma_{A_{0}}, \sigma_{B}\right)$ for which hypotheses of Theorem 1.16 hold true. This set is not empty.

Remark 1.19. Let us compare the hypotheses of Theorem 1.16 with those of the existence theorem we have proved in [3]. For that we consider the model case where

$$
H(x, \xi)=\left(1+|\xi|^{2}\right)^{\lambda / 2}, \quad \lambda \geq 0
$$

for which it is easy to see that (1.9) holds with $b=$ constant, $r=\infty, A_{0}=1, \sigma_{A_{0}}=(\lambda-1)^{+}, B=1$ and $\sigma_{B}=0$. In this case, Theorem 1.16 implies the uniqueness of the renormalized solution when

$$
(\lambda-1)^{+}<\sigma^{*}(N, p, \infty)=\frac{2(p-1)}{N-1}
$$

i.e.

$$
0 \leq \lambda<\frac{2 p+N-3}{N-1}
$$

On the other hand, we proved in [3] that there exists a renormalized solution when

$$
0 \leq \lambda \leq p-1
$$

But when (1.39) holds, one has

$$
p-1 \geq \frac{2 p+N-3}{N-1}
$$

(with a strict inequality when $p>\frac{2 N-4}{N-3}$ ), and therefore we have proved existence for $\lambda$ in the interval in which Theorem 1.16 implies uniqueness, but also in the interval $\frac{2 p+N-3}{N-1} \leq \lambda \leq p-1$, in which case uniqueness is not proved. 


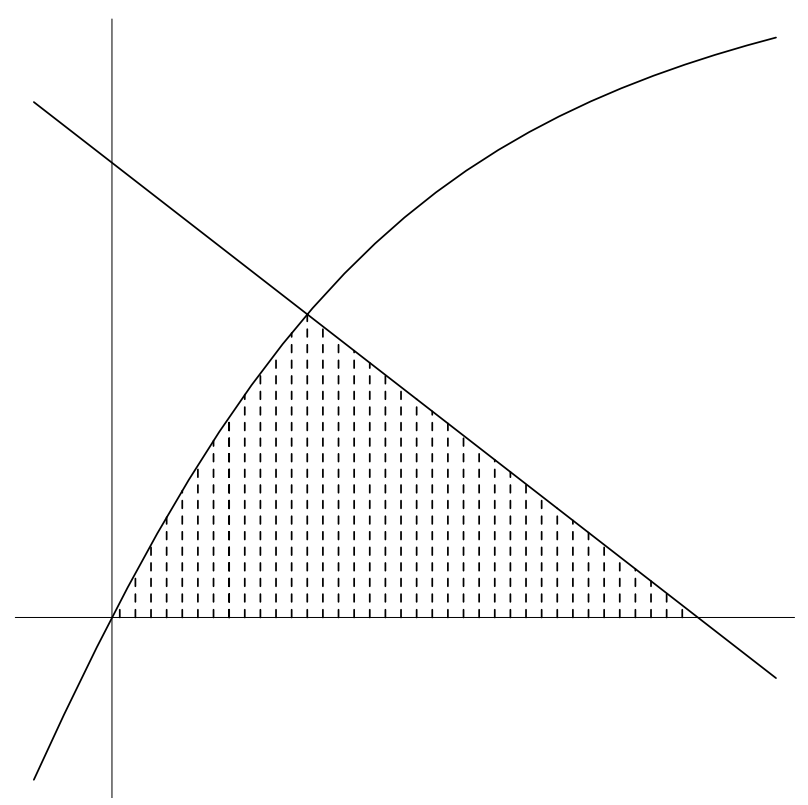

Figure 3. Values of $\left(\sigma_{A_{0}}, \sigma_{B}\right)$ for which Theorem 1.16 holds true. The horizontal axis gives the values of $\sigma_{A_{0}}$ and the vertical axis the values of $\sigma_{B}$. Theorem 1.16 holds true when the values of $\left(\sigma_{A_{0}}, \sigma_{B}\right)$ are inside the curvilinear triangle defined by the horizontal axis $\sigma_{B}=0$, the oblique line $\sigma_{A_{0}}+\sigma_{B}=\sigma^{*}$, and the branch of the hyperbola $\left(\sigma_{B}-(p-1)\right)^{2}-\left(\sigma_{A_{0}}-\frac{p-1}{N-1}\right)^{2}=$ $(p-1)^{2}-\left(\frac{p-1}{N-1}\right)^{2}$ which contains the origin.

Remark 1.20. In this remark we consider the case where $p=\frac{2 N-4}{N-3}$, which is a bordeline case between Theorems 1.11 and 1.16.

There is a discrepancy between these two Theorems in this limit case. In Theorem $1.11, \sigma^{*}(N, p, r)$ is defined for $2<p<\frac{2 N-4}{N-3}$, while in Theorem 1.16, $\sigma^{*}(N, p, r)$ is defined for $\frac{2 N-4}{N-3} \leq p<N$. But when $p<\frac{2 N-4}{N-3}$ tends to $\frac{2 N-4}{N-3}$, formulas $(1.33)$ and $(1.36)$ show that

$$
\begin{gathered}
r \rightarrow+\infty \\
\sigma^{*}(N, p, r) \rightarrow \frac{2}{N-3}, \\
\rho^{*}(N, p) \rightarrow \frac{2}{N-3} .
\end{gathered}
$$

Therefore the set of admissible values defined by Theorem 1.11 tends to the line segment

$$
\left\{\left(\sigma_{A_{0}}, \sigma_{B}\right): 0<\sigma_{A_{0}}<\frac{2}{N-3}, \sigma_{B}=0\right\}
$$

when $p$ tends to $\frac{2 N-4}{N-3}$. In contrast, the set of admissible values defined by Theorem 1.16 for $p=\frac{2 N-4}{N-3}$ is a curvilinear triangle, and therefore the admissible sets are very different. The sole matching point is the fact that the basis of the curvilinear triangle of Theorem 1.16 is the line segment

$$
\left\{\left(\sigma_{A_{0}}, \sigma_{B}\right): 0<\sigma_{A_{0}}<\sigma^{*}\left(N, \frac{2 N-4}{N-3}, r\right)=\frac{2}{N-3}-\frac{2 N}{N-3} \frac{1}{r}, \sigma_{B}=0\right\}
$$


which coincides with the line segment defined above when $r=+\infty$. Fortunately this covers the case of the model example $H(x, \xi)=\left(1+|\xi|^{2}\right)^{\lambda / 2}, \lambda \geq 0$, considered in Remarks 1.14 and 1.19 since $\frac{(N+1) p-2}{2(N-1)}=\frac{2 p+N-3}{N-1}$ when $p=\frac{2 N-4}{N-3}$.

\subsection{Final remarks}

Remark 1.21. Roughly speaking, the hypotheses which we assume in the uniqueness Theorems $1.7,1.11$ and 1.16 are the strong monotonicity of $a$, the local Lipschitz continuity of $H$, the monotonicity of $G$, and the fact that $f \in L^{1}(\Omega)$; while in [3] we proved an existence result under the more general assumptions that $a$ defines a pseudo-monotone operator, $H$ and $G$ satisfies natural growth conditions, $G$ satisfies a sign condition, and $f$ is a Radon measure with bounded total variation. Unfortunately, we were not able to prove an uniqueness result in such a generality and we had to make further strong restrictions. However, this is not due to the fact that the right-hand side is a measure, since even in the case where the right-hand side is an element of the dual space $W^{-1, p^{\prime}}(\Omega)$ (and where the solution is an usual weak solution), we have to make analogous strong restrictions in order to obtain uniqueness results (see [4]).

Remark 1.22. Theorems 1.7, 1.11 and 1.16 prove the uniqueness of the renormalized solution of (1.4) under suitable hypotheses. As usual, such uniqueness results imply some continuity result.

Consider, under the hypotheses of Theorem 1.7, 1.11 or 1.16 , the unique renormalized solution $u_{\epsilon}$ of (1.4) corresponding to the right-hand side

$$
f_{\varepsilon}-\operatorname{div}\left(g_{\varepsilon}\right)
$$

and assume that

$$
\begin{gathered}
f_{\varepsilon} \rightarrow f \text { in } L^{1}(\Omega) \text { weakly, } \\
g_{\varepsilon} \rightarrow g \text { in }\left(L^{p^{\prime}}(\Omega)\right)^{N} \text { weakly. }
\end{gathered}
$$

We also assume that, further to the uniqueness hypotheses, one has

$$
\sigma_{A_{0}}+\sigma_{B}+1 \leq p-1
$$

which in particular implies that

$$
|H(x, \xi)| \leq b(x)\left(B(x)+A_{0}+|\xi|\right)^{p-1}+|H(x, 0)|,
$$

where $b \in L^{N, 1}(\Omega), A_{0} \in \mathbb{R}, B^{p-1} \in L^{N^{\prime}, \infty}(\Omega)$ and $H(x, 0) \in L^{1}(\Omega)$. Moreover we assume that, for some $\rho$ with $0 \leq \rho<\frac{N(p-1)}{N-p}$, one has

$$
\left\{\begin{array}{l}
|G(x, s)| \leq b_{2}(x)|s|^{\rho}+b_{3}(x), \\
b_{2} \in L^{z^{\prime}, 1}(\Omega), b_{3} \in L^{1}(\Omega),
\end{array}\right.
$$

for almost every $x \in \Omega$ and for every $s \in \mathbb{R}$, where

$$
z=\frac{N(p-1)}{N-p} \frac{1}{\rho}, \quad \frac{1}{z}+\frac{1}{z^{\prime}}=1 .
$$

Therefore the hypotheses under which existence is proved in [3] are satisfied and there exists a unique renormalized solution of (1.4). Moreover the existence proof of [3] also proves that

$$
T_{k}\left(u_{\varepsilon}\right) \text { is bounded in } W_{0}^{1, p}(\Omega) .
$$


A proof which is very similar to the proof of the existence result of [3] then shows that

$$
T_{k}\left(u_{\varepsilon}\right) \rightarrow T_{k}(u) \text { in } W_{0}^{1, p}(\Omega) \text { strongly, }
$$

where $u$ is the unique renormalized solution of (1.4) corresponding to the right-hand side $f-\operatorname{div}(g)$.

\section{Proofs of the Uniqueness Results}

\subsection{Proof of Theorem $\mathbf{1 . 7}$}

\section{Preliminary remark}

In the steps 1 to 4 below, we will prove the uniqueness of a renormalized solution of (1.4) under assumptions (1.5)-(1.11), (1.25), (1.26), (1.29) and

$$
\left\{\begin{array}{l}
\sigma_{A_{0}}=0 \\
0 \leq \sigma_{B}<\sigma^{*}(N, p, r)
\end{array}\right.
$$

This is sufficient to prove Theorem 1.7. Indeed, when $\sigma_{A_{0}}$ and $\sigma_{B}$ satisfy (1.27) and (1.28), we can reconduce ourselves to the case (2.1), since every function $H$ which satisfies

$$
|H(x, \xi)-H(x, \eta)| \leq b(x)\left(A_{0}+|\xi|+|\eta|\right)^{\sigma_{A_{0}}}(B(x)+|\xi|+|\eta|)^{\sigma_{B}}|\xi-\eta|
$$

also satisfies

$$
|H(x, \xi)-H(x, \eta)| \leq b(x)\left(A_{0}+B(x)+|\xi|+|\eta|\right)^{\sigma_{A_{0}}+\sigma_{B}}|\xi-\eta|
$$

and taking $\bar{\sigma}_{A_{0}}=0, \bar{\sigma}_{B}=\sigma_{A_{0}}+\sigma_{B}$ and $\bar{B}(x)=A_{0}+B(x)$ we are reconduced to (2.1).

First step. Observe that under the assumptions of Theorem 1.7, and more specifically using (1.8), (1.9), (1.16), $(1.26),(1.28)$ (or $(2.1))$, and (1.29)

$$
\text { every renormalized solution of (1.4) satisfies } H(x, \nabla u) \in L^{1}(\Omega) \text {. }
$$

Observe also that Theorem 1.7 is concerned with the case $p \leq 2$, but that from now on, the proof made in this first step will be exactly the same also when $p>2$, since in this step we will no more use the hypothesis $p \leq 2$ but only (2.2).

Define for $m>0$ the "remainder" $S_{m}$ of the truncation $T_{m}$, that is

$$
S_{m}(s)=s-T_{m}(s) \quad \forall s \in \mathbb{R},
$$

or in other terms

$$
S_{m}(s)= \begin{cases}0 & |s| \leq m \\ (|s|-m) \operatorname{sign}(s) & |s|>m\end{cases}
$$

and recall the definition (1.22) of the function $h_{n}$. Since $u_{1}$ and $u_{2}$ are renormalized solutions of (1.4), the functions

$$
\left\{\begin{array}{l}
v_{1}=h_{n}\left(u_{2}\right) T_{k}\left(S_{m}\left(T_{2 n}\left(u_{1}\right)-T_{2 n}\left(u_{2}\right)\right)\right) \\
v_{2}=h_{n}\left(u_{1}\right) T_{k}\left(S_{m}\left(T_{2 n}\left(u_{1}\right)-T_{2 n}\left(u_{2}\right)\right)\right)
\end{array}\right.
$$

belong to $W_{0}^{1, p}(\Omega) \cap L^{\infty}(\Omega)$. We can therefore choose $v=v_{1}$ and $h=h_{n}$ in the equation (1.18) (or more exactly (1.19)) satisfied by $u_{1}$, and $v=v_{2}$ and $h=h_{n}$ in the equation (1.18) satisfied by $u_{2}$. We obtain by 
difference

$$
\begin{aligned}
\int_{\Omega}\left[a\left(x, \nabla u_{1}\right)\right. & \left.-a\left(x, \nabla u_{2}\right)\right]\left(\nabla u_{1}-\nabla u_{2}\right) \chi_{\left\{\left|S_{m}\left(u_{1}-u_{2}\right)\right|<k\right\}} h_{n}\left(u_{1}\right) h_{n}\left(u_{2}\right) \\
& +\int_{\Omega} a\left(x, \nabla u_{1}\right) \nabla u_{1} h_{n}^{\prime}\left(u_{1}\right) h_{n}\left(u_{2}\right) T_{k}\left(S_{m}\left(u_{1}-u_{2}\right)\right) \\
& -\int_{\Omega} a\left(x, \nabla u_{2}\right) \nabla u_{2} h_{n}\left(u_{1}\right) h_{n}^{\prime}\left(u_{2}\right) T_{k}\left(S_{m}\left(u_{1}-u_{2}\right)\right) \\
& +\int_{\Omega} a\left(x, \nabla u_{1}\right) \nabla u_{2} h_{n}\left(u_{1}\right) h_{n}^{\prime}\left(u_{2}\right) T_{k}\left(S_{m}\left(u_{1}-u_{2}\right)\right) \\
& -\int_{\Omega} a\left(x, \nabla u_{2}\right) \nabla u_{1} h_{n}^{\prime}\left(u_{1}\right) h_{n}\left(u_{2}\right) T_{k}\left(S_{m}\left(u_{1}-u_{2}\right)\right) \\
& +\int_{\Omega}\left[G\left(x, u_{1}\right)-G\left(x, u_{2}\right)\right] h_{n}\left(u_{1}\right) h_{n}\left(u_{2}\right) T_{k}\left(S_{m}\left(u_{1}-u_{2}\right)\right) \\
= & -\int_{\Omega}\left[H\left(x, \nabla u_{1}\right)-H\left(x, \nabla u_{2}\right)\right] h_{n}\left(u_{1}\right) h_{n}\left(u_{2}\right) T_{k}\left(S_{m}\left(u_{1}-u_{2}\right)\right),
\end{aligned}
$$

where, by an abuse of notation, we wrote $u_{1}$ and $u_{2}$ in place of $T_{2 n}\left(u_{1}\right)$ and $T_{2 n}\left(u_{2}\right)$.

We now let $n$ tend to infinity for fixed $k$ and $m$.

Since

$$
\left[a\left(x, \nabla u_{1}\right)-a\left(x, \nabla u_{2}\right)\right]\left(\nabla u_{1}-\nabla u_{2}\right) \chi_{\left\{\left|S_{m}\left(u_{1}-u_{2}\right)\right|<k\right\}}
$$

is a measurable function which is non negative, and since $h_{n}\left(u_{1}\right) h_{n}\left(u_{2}\right)$ tends to 1 a.e. in $\Omega$, Fatou lemma implies that

$$
\begin{aligned}
& \int_{\Omega}\left[a\left(x, \nabla u_{1}\right)-a\left(x, \nabla u_{2}\right)\right]\left(\nabla u_{1}-\nabla u_{2}\right) \chi_{\left\{\left|S_{m}\left(u_{1}-u_{2}\right)\right|<k\right\}} \\
& \leq \liminf _{n} \int_{\Omega}\left[a\left(x, \nabla u_{1}\right)-a\left(x, \nabla u_{2}\right)\right]\left(\nabla u_{1}-\nabla u_{2}\right) \chi_{\left\{\left|S_{m}\left(u_{1}-u_{2}\right)\right|<k\right\}} h_{n}\left(u_{1}\right) h_{n}\left(u_{2}\right) .
\end{aligned}
$$

In view of the definition (1.23) of $h_{n}$, the absolute value of the second term of (2.5) is easily estimated by

$$
\frac{k}{n} \int_{n<\left|u_{1}\right|<2 n} a\left(x, \nabla u_{1}\right) \nabla u_{1}
$$

which tends to 0 by (1.17).

Similarly, the third term of (2.5) tends to zero.

In view of the growth condition (1.6) on $a$, the absolute value of the fourth term of (2.5) is estimated by

$$
\begin{array}{r}
\frac{k}{n} c \int_{\left\{n<\left|u_{2}\right|<2 n\right\} \cap\left\{\left|u_{1}\right| \leq 2 n\right\}}\left[\left|\nabla u_{1}\right|^{p-1}+a_{0}(x)\right]\left|\nabla u_{2}\right| \leq k c\left[\left(\frac{1}{n} \int_{\left\{\left|u_{1}\right| \leq 2 n\right\}}\left|\nabla u_{1}\right|^{p}\right)^{\frac{1}{p^{\prime}}}\left(\frac{1}{n} \int_{\left\{n<\left|u_{2}\right|<2 n\right\}}\left|\nabla u_{2}\right|^{p}\right)^{\frac{1}{p}}\right. \\
\left.+\left(\frac{1}{n} \int_{\Omega}\left|a_{0}\right|^{p^{\prime}}\right)^{\frac{1}{p^{\prime}}}\left(\frac{1}{n} \int_{\left\{n<\left|u_{2}\right|<2 n\right\}}\left|\nabla u_{2}\right|^{p}\right)^{\frac{1}{p}}\right]
\end{array}
$$

which tends to 0 since

$$
\frac{1}{n} \int_{\left\{n<\left|u_{2}\right|<2 n\right\}}\left|\nabla u_{2}\right|^{p} \rightarrow 0
$$


by (1.17), and (1.5), while

$$
\frac{1}{n} \int_{\left\{\left|u_{1}\right|<2 n\right\}}\left|\nabla u_{1}\right|^{p} \rightarrow 0
$$

indeed (2.6) can be easily proved by using in (1.18) the test function $v=T_{2 n}\left(u_{1}\right)$ and $h=h_{j}$, and letting $j$ tend to infinity for $n$ fixed.

Similarly the fifth term in (2.5) tends to zero.

In view of hypothesis (1.10), the last term of the left-hand side of (2.5) is non negative.

Finally, since $H\left(x, \nabla u_{1}\right)$ and $H\left(x, \nabla u_{2}\right)$ are in $L^{1}(\Omega)$ thanks to $(2.2)$, Lebesgue dominated convergence theorem implies that the right-hand side of (2.5) tends to

$$
\int_{\Omega}\left[H\left(x, \nabla u_{1}\right)-H\left(x, \nabla u_{2}\right)\right] T_{k}\left(S_{m}\left(u_{1}-u_{2}\right)\right),
$$

the absolute value of which is estimated by

$$
k \int_{\Omega}\left|H\left(x, \nabla u_{1}\right)-H\left(x, \nabla u_{2}\right)\right| \chi_{\left\{\left|u_{1}-u_{2}\right|>m\right\}} .
$$

Passing to the limit in $n$ in (2.5), we have proved that

$$
\int_{\Omega}\left[a\left(x, \nabla u_{1}\right)-a\left(x, \nabla u_{2}\right)\right]\left(\nabla u_{1}-\nabla u_{2}\right) \chi_{\left\{\left|S_{m}\left(u_{1}-u_{2}\right)\right|<k\right\}} \leq k \int_{\Omega}\left|H\left(x, \nabla u_{1}\right)-H\left(x, \nabla u_{2}\right)\right| \chi_{\left\{\left|u_{1}-u_{2}\right|>m\right\}} .
$$

Up to this point, we used hypothesis $p \leq 2$ only to say that $H\left(x, \nabla u_{1}\right)$ and $H\left(x, \nabla u_{2}\right)$ are in $L^{1}(\Omega)$. Let us explicitly observe that, when $p>2$, hypotheses on $\sigma_{A_{0}}$ and $\sigma_{B}$ made in Theorem 1.11 and Theorem 1.16 will again insure that $H\left(x, \nabla u_{1}\right)$ and $H\left(x, \nabla u_{2}\right)$ are in $L^{1}(\Omega)$. Therefore (2.7) also holds for $p>2$.

Second step. Using hypotheses (1.7), (1.9), and (2.1), we deduce from (2.7) that

$$
\beta \int_{\Omega} \frac{\left|\nabla u_{1}-\nabla u_{2}\right|^{2}}{\left(A(x)+\left|\nabla u_{1}\right|+\left|\nabla u_{2}\right|\right)^{2-p}} \chi_{\left\{\left|S_{m}\left(u_{1}-u_{2}\right)\right|<k\right\}} \leq k \int_{\Omega} b(x)\left(B(x)+\left|\nabla u_{1}\right|+\left|\nabla u_{2}\right|\right)^{\sigma_{B}}\left|\nabla u_{1}-\nabla u_{2}\right| \chi_{\left\{\left|u_{1}-u_{2}\right|>m\right\}} .
$$

We now consider some $s$ such that

$$
1 \leq s<\frac{2 N(p-1)}{N-(2-p)}=s_{*}
$$

we will actually choose $s$ very close to $\frac{2 N(p-1)}{N-(2-p)}$. Observe that such a number $s$ exists in view of hypothesis (1.24), and that $s<2$ since $p \leq 2$. Using Hölder inequality with the exponent $\frac{2}{s}$, we obtain

$$
\begin{aligned}
\int_{\Omega}\left|\nabla T_{k}\left(S_{m}\left(u_{1}-u_{2}\right)\right)\right|^{s} & =\int_{\Omega} \frac{\left|\nabla T_{k}\left(S_{m}\left(u_{1}-u_{2}\right)\right)\right|^{s}}{\left(A(x)+\left|\nabla u_{1}\right|+\left|\nabla u_{2}\right|\right)^{(2-p) \frac{s}{2}}}\left(A(x)+\left|\nabla u_{1}\right|+\left|\nabla u_{2}\right|\right)^{(2-p) \frac{s}{2}} \\
& \leq\left(\int_{\Omega} \frac{\left|\nabla T_{k}\left(S_{m}\left(u_{1}-u_{2}\right)\right)\right|^{2}}{\left(A(x)+\left|\nabla u_{1}\right|+\left|\nabla u_{2}\right|\right)^{2-p}}\right)^{\frac{s}{2}}\left(\int_{\Omega}\left(A(x)+\left|\nabla u_{1}\right|+\left|\nabla u_{2}\right|\right)^{\frac{s(2-p)}{2-s}}\right)^{\frac{2-s}{2}} .
\end{aligned}
$$

From (2.8) we obtain

$$
\int_{\Omega}\left|\nabla T_{k}\left(S_{m}\left(u_{1}-u_{2}\right)\right)\right|^{s} \leq M k^{\frac{s}{2}},
$$


where $M$ is given by

$$
M=\frac{1}{\beta^{\frac{s}{2}}}\left(\int_{\left\{\left|u_{1}-u_{2}\right|>m\right\}} b(x)\left(B(x)+\left|\nabla u_{1}\right|+\left|\nabla u_{2}\right|\right)^{\sigma_{B}}\left|\nabla u_{1}-\nabla u_{2}\right|\right)^{\frac{s}{2}}\left(\int_{\Omega}\left(A(x)+\left|\nabla u_{1}\right|+\left|\nabla u_{2}\right|\right)^{\frac{s(2-p)}{2-s}}\right)^{\frac{2-s}{2}} .
$$

From (2.10) and Lemma 4.1 of Appendix 2 below with $\theta=\frac{s}{2}$, we deduce that

$$
\left\|\left|\nabla S_{m}\left(u_{1}-u_{2}\right)\right|^{\frac{s}{2}} \mid\right\|_{L^{\frac{N}{N-\frac{s}{2}}, \infty}(\Omega)} \leq C(N, s) M
$$

which implies, when $q$ satisfies

$$
1 \leq q<\frac{N}{N-\frac{s}{2}}
$$

that

$$
\left\|\left|\nabla S_{m}\left(u_{1}-u_{2}\right)\right|^{\frac{s}{2}} \mid\right\|_{L^{q}(\Omega)} \leq C(N, s, q,|\Omega|) M,
$$

where $C(N, s, q,|\Omega|)$ is a constant depending only on $N, s, q$ and $|\Omega|$.

Since $s$ satisfies (2.9), a simple calculation shows that (2.12) is equivalent to

$$
1 \leq q<\frac{N-(2-p)}{N-1}=q_{*}
$$

Third step. In this step, we estimate $M$.

As in the proof of the existence Theorem in [3], we introduce a set $Z$ in the following way: since $|\Omega|$ is finite, the set of the constants such that $\left|\left\{x \in \Omega,\left|u_{1}(x)-u_{2}(x)\right|=c\right\}\right|>0$ is at most countable. Let $Z \subset \Omega$ be the union of those sets such that $\left|\left\{x \in \Omega,\left|u_{1}(x)-u_{2}(x)\right|=c\right\}\right|=0$; its complementary $Z^{c}=\Omega-Z$ is therefore the countable set, union of those sets such that $\left|\left\{x \in \Omega,\left|u_{1}(x)-u_{2}(x)\right|=c\right\}\right|>0$. Since for every $c$

$$
\nabla\left(u_{1}-u_{2}\right)=0 \quad \text { a.e. on }\left\{x \in \Omega,\left|u_{1}(x)-u_{2}(x)\right|=c\right\},
$$

we obtain that

$$
\nabla\left(u_{1}-u_{2}\right)=0 \quad \text { a.e. on } Z^{c} .
$$

Using Hölder inequality in the first integral of (2.11), we have

$$
\begin{aligned}
\int_{\left\{\left|u_{1}-u_{2}\right|>m\right\}} b(x)\left(B(x)+\left|\nabla u_{1}\right|+\left|\nabla u_{2}\right|\right)^{\sigma_{B}}\left|\nabla u_{1}-\nabla u_{2}\right| \\
=\int_{\left\{\left|u_{1}-u_{2}\right|>m\right\} \cap Z} b(x)\left(B(x)+\left|\nabla u_{1}\right|+\left|\nabla u_{2}\right|\right)^{\sigma_{B}}\left|\nabla S_{m}\left(u_{1}-u_{2}\right)\right|^{\frac{s}{2} \frac{2}{s}} \\
\leq\|b\|_{L^{r}\left(\left\{\left|u_{1}-u_{2}\right|>m\right\} \cap Z\right)}\left\|B+\left|\nabla u_{1}\right|+\left|\nabla u_{2}\right|\right\|_{L^{\rho}(\Omega)}^{\sigma_{B}} \\
\times\left\|\left|\nabla S_{m}\left(u_{1}-u_{2}\right)\right|^{\frac{s}{2}}\right\|_{L^{q}(\Omega)}^{\frac{2}{s}}|\Omega|^{\frac{1}{t}}
\end{aligned}
$$

whenever we are able to find $\rho, s, q$ and $t$ such that

$$
\frac{1}{r}+\frac{\sigma_{B}}{\rho}+\frac{2}{s q}+\frac{1}{t}=1
$$




$$
\begin{gathered}
1 \leq \rho<\frac{N(p-1)}{N-1}=\rho_{*}, \\
1 \leq s<\frac{2 N(p-1)}{N-(2-p)}=s_{*}, \\
1<t \leq+\infty
\end{gathered}
$$

where $q$ satisfies (2.14) (some of those requirements are dictated by previous hypotheses).

Setting

$$
F(\rho, s, q)=\frac{1}{r}+\frac{\sigma_{B}}{\rho}+\frac{2}{s q}-1,
$$

we observe that hypothesis $(2.1)$ reads as $F\left(\rho_{*}, s_{*}, q_{*}\right)<0$, so that we can choose $\rho$ with $1<\rho<\rho_{*}$ and close to $\rho_{*}$ such that

$$
F\left(\rho, s_{*}, q_{*}\right)<0 .
$$

We now choose $s$ with $1<s<s_{*}$ and close to $s_{*}$ such that

$$
F\left(\rho, s, q_{*}\right)<0
$$

Finally, we choose $q$ with $1<q<q_{*}$ and close to $q_{*}$ such that

$$
F(\rho, s, q)<0
$$

From now on we consider these values of $\rho, s$ and $q$ as fixed; observe that (2.14), (2.18), and (2.19) are satisfied. Setting $F(\rho, s, q)=-\frac{1}{t}$ defines $t$ which satisfies (2.20) and (2.17). Therefore the above use of Hölder inequality is licit.

We explicitly observe that for these values of $\rho, s, q$ and $t, M$ is a finite number, which implies that the left-hand side of (2.13) is finite.

Fourth step. From (2.13), (2.11), and (2.16) we deduce that

$$
\begin{aligned}
\left\|\left|\nabla S_{m}\left(u_{1}-u_{2}\right)\right|^{\frac{s}{2}}\left|\left\|_{L^{q}(\Omega)} \leq \frac{C(N, s, q,|\Omega|)}{\beta^{\frac{s}{2}}}\right\| b\left\|_{L^{r}\left(\left\{\left|u_{1}-u_{2}\right|>m\right\} \cap Z\right)}^{\frac{s}{2}}\right\| B+\right| \nabla u_{1}|+| \nabla u_{2} \mid\right\|_{L^{\rho}(\Omega)}^{\sigma_{B}(\Omega)} \\
\quad \times\left\|\left|\nabla S_{m}\left(u_{1}-u_{2}\right)\right|^{\frac{s}{2}}\right\|_{L^{q}(\Omega)}\left(\int_{\Omega}\left(A(x)+\left|\nabla u_{1}\right|+\left|\nabla u_{2}\right|\right)^{\frac{s(2-p)}{2-s}}\right)^{\frac{2-s}{2}}
\end{aligned}
$$

Since every term in this inequality is finite, we obtain that

$$
\left(1-C_{0}\|b\|_{L^{r}\left(\left\{\left|u_{1}-u_{2}\right|>m\right\} \cap Z\right)}^{\frac{s}{2}}\right)\left\|\left|\nabla S_{m}\left(u_{1}-u_{2}\right)\right|^{\frac{s}{2}} \mid\right\|_{L^{q}(\Omega)} \leq 0,
$$

where $C_{0}$ is a constant which depends only on $\left|\nabla u_{1}\right|,\left|\nabla u_{2}\right|, A, B, \sigma_{B}, \beta, p, N,|\Omega|, s, q$ and $\rho$, and will therefore be considered as fixed; in particular this constant does not depend on $m$.

We want to deduce from $(2.21)$ that $u_{1}-u_{2}=0$. We argue by contradiction, assuming that $u_{1}-u_{2} \neq 0$.

In view of the definition of $Z$ (see third step above), the function

$$
m \rightarrow\|b\|_{L^{r}\left(\left\{\left|u_{1}-u_{2}\right|>m\right\} \cap Z\right)}^{\frac{s}{2}}
$$


is a continuous function which tends to 0 when $m$ tends to infinity and to $\|b\|_{L^{r}(Z)}^{\frac{s}{2}}>0$ when $m$ tends to zero. Therefore either

$$
C_{0}\|b\|_{L^{r}(Z)}^{\frac{s}{2}} \leq \frac{1}{2}
$$

and (2.21) with $m=0$ implies that $S_{0}\left(u_{1}-u_{2}\right)=0$, a contradiction; or

$$
C_{0}\|b\|_{L^{r}(Z)}^{\frac{s}{2}}>\frac{1}{2}
$$

and we can choose $m=m_{1}>0$ such that

$$
C_{0}\|b\|_{L^{r}\left(\left\{\left|u_{1}-u_{2}\right|>m_{1}\right\} \cap Z\right)}^{\frac{s}{2}}=\frac{1}{2} .
$$

Then (2.21) implies that $S_{m_{1}}\left(u_{1}-u_{2}\right)=0$, i.e. $\left|u_{1}-u_{2}\right| \leq m_{1}$ a.e., a contradiction with (2.22).

This completes the proof of Theorem 1.7.

The proof of the comparison result stated in Remark 1.8 is very similar: it is indeed sufficient to use the test function $T_{k}\left(S_{m}\left(T_{2 n}\left(u_{1}\right)-T_{2 n}\left(u_{2}\right)\right)^{+}\right)$in place of $T_{k}\left(S_{m}\left(T_{2 n}\left(u_{1}\right)-T_{2 n}\left(u_{2}\right)\right)\right)$ in $(2.4)$ in the above proof.

\subsection{Proof of Theorem $\mathbf{1 . 1 1}$}

Preliminary remark. In the steps 1 to 4 below, we will prove the uniqueness of a renormalized solution of (1.4) under assumptions (1.5)-(1.11), (1.32), (1.33), (1.36), and

$$
\begin{gathered}
\rho^{*}(N, p)<\sigma_{A_{0}}<\sigma^{*}(N, p, r), \\
0 \leq \sigma_{B}<\sigma^{*}(N, p, r)-\sigma_{A_{0}} .
\end{gathered}
$$

This is sufficient to prove Theorem 1.11. Indeed, when $\sigma_{A_{0}}$ and $\sigma_{B}$ satisfy (1.34) and (1.35), either $\sigma_{A_{0}}$ satisfies (2.23) or $\sigma_{A_{0}}$ satisfies

$$
0 \leq \sigma_{A_{0}}<\rho^{*}
$$

In the first case, i.e. when (2.23) holds true, (2.24) is equivalent to (1.35) (see Fig. 2) and Theorem 1.11 will be proved in steps 1 to 4 below. In the second case, i.e. when (2.25) holds true, (1.35) is equivalent to

$$
0 \leq \sigma_{B}<\sigma^{*}(N, p, r)-\rho^{*}(N, p)
$$

(see again Fig. 2), and we will reconduce ourselves to the first case. Indeed when a function $H$ satisfies

$$
|H(x, \xi)-H(x, \eta)| \leq b(x)\left(A_{0}+|\xi|+|\eta|\right)^{\sigma_{A_{0}}}(B(x)+|\xi|+|\eta|)^{\sigma_{B}}|\xi-\eta|,
$$

with (1.32), i.e. $A_{0}>0$, it also satisfies

$$
|H(x, \xi)-H(x, \eta)| \leq \frac{b(x)}{A_{0}^{\delta}}\left(A_{0}+|\xi|+|\eta|\right)^{\sigma_{A_{0}}+\delta}(B(x)+|\xi|+|\eta|)^{\sigma_{B}}|\xi-\eta|,
$$

for every $\delta \geq 0$, and taking $\bar{\sigma}_{A_{0}}=\sigma_{A_{0}}+\delta$ and $\bar{\sigma}_{B}=\sigma_{B}$, we are reconduced to the case where (2.23) and (2.24) hold true if we can choose some $\delta \geq 0$ such that

$$
\left\{\begin{array}{l}
\rho^{*}(N, p)<\bar{\sigma}_{A_{0}}=\sigma_{A_{0}}+\delta<\sigma^{*}(N, p, r), \\
0 \leq \bar{\sigma}_{B}=\sigma_{B}<\sigma^{*}(N, p, r)-\bar{\sigma}_{A_{0}}=\sigma^{*}(N, p, r)-\sigma_{A_{0}}-\delta .
\end{array}\right.
$$


But (2.27) is equivalent to

$$
\left\{\begin{array}{l}
\rho^{*}(N, p)-\sigma_{A_{0}}<\delta<\sigma^{*}(N, p, r)-\sigma_{A_{0}} \\
0 \leq \sigma_{B} \\
\delta<\sigma^{*}(N, p, r)-\sigma_{B}-\sigma_{A_{0}}
\end{array}\right.
$$

i.e. to

$$
\left\{\begin{array}{l}
0 \leq \sigma_{B} \\
\rho^{*}(N, p)-\sigma_{A_{0}}<\delta<\sigma^{*}(N, p, r)-\sigma_{B}-\sigma_{A_{0}}
\end{array}\right.
$$

and choosing some $\delta>0$ satisfying (2.28) is possible when (2.25) and (2.26) hold true.

First step. Observe that $H\left(x, \nabla u_{1}\right)$ and $H\left(x, \nabla u_{2}\right)$ belong to $L^{1}(\Omega)$ thanks to (1.8), (1.9), (1.16), (1.33), (1.36), and (2.23). But, as said at the beginning of the proof of Theorem 1.7, the first step of its proof holds also for $p>2$, whenever $H\left(x, \nabla u_{1}\right)$ and $H\left(x, \nabla u_{2}\right)$ belong to $L^{1}(\Omega)$, therefore (2.7) holds in the present case.

Second step. Using hypotheses (1.7), (1.9), and (1.32), we deduce from (2.7) that

$$
\begin{aligned}
& \int_{\Omega}\left(A_{0}+\left|\nabla u_{1}\right|+\left|\nabla u_{2}\right|\right)^{p-2}\left|\nabla T_{k}\left(S_{m}\left(u_{1}-u_{2}\right)\right)\right|^{2} \\
& =\int_{\Omega}\left(A_{0}+\left|\nabla u_{1}\right|+\left|\nabla u_{2}\right|\right)^{p-2}\left|\nabla u_{1}-\nabla u_{2}\right|^{2} \chi_{\left\{\left|S_{m}\left(u_{1}-u_{2}\right)\right|<k\right\}} \leq M k,
\end{aligned}
$$

where $M$ is given by

$$
M=\frac{1}{\beta} \int_{\left\{\left|u_{1}-u_{2}\right|>m\right\}} b(x)\left(A_{0}+\left|\nabla u_{1}\right|+\left|\nabla u_{2}\right|\right)^{\sigma_{A_{0}}}\left(B(x)+\left|\nabla u_{1}\right|+\left|\nabla u_{2}\right|\right)^{\sigma_{B}}\left|\nabla u_{1}-\nabla u_{2}\right| .
$$

We now apply Lemma 4.2 with $v=\left(A_{0}+\left|\nabla u_{1}\right|+\left|\nabla u_{2}\right|\right)^{p-2}$. Observe that $v \geq A_{0}>0$ a.e. in $\Omega$ and that $v \in L^{s}(\Omega)$ with $s<\frac{N}{N-1} \frac{p-1}{p-2}$; since $\frac{N}{N-1} \frac{p-1}{p-2}>\frac{N}{2}$ when $p$ satisfies (1.31), we can apply Lemma 4.2 with $s$ and $\gamma$ such that

$$
\left\{\begin{array}{l}
\frac{N}{2}<s<\frac{N(p-1)}{(N-1)(p-2)} \\
1<\gamma<\frac{2 N(s-1)}{2(N-1) s-N}
\end{array}\right.
$$

A simple calculation shows that the second line of (2.31) is equivalent to

$$
1<\gamma<\frac{2(N+p-2)}{p(N-1)}=\gamma_{*}
$$

We deduce from (2.29) and Lemma 4.2 that

$$
\int_{\Omega}\left(A_{0}+\left|\nabla u_{1}\right|+\left|\nabla u_{2}\right|\right)^{p-2}\left|\nabla S_{m}\left(u_{1}-u_{2}\right)\right|^{\gamma} \leq C_{0} M^{\gamma},
$$

where $C_{0}$ is a constant which only depends on $N, s, \gamma, A_{0},\left\|\left|\nabla u_{1}\right|^{p-2}\right\|_{L^{s}(\Omega)}$ and $\left\|\left|\nabla u_{2}\right|\right\|_{L^{s}(\Omega)}$.

Third step. In this step, we estimate $M$.

As in the proof of Theorem 1.7, we introduce a set $Z$ in the following way: since $|\Omega|$ is finite, the set of the constants such that $\left|\left\{x \in \Omega,\left|u_{1}(x)-u_{2}(x)\right|=c\right\}\right|>0$ is at most countable. Let $Z \subset \Omega$ be the union of those 
sets such that $\left|\left\{x \in \Omega,\left|u_{1}(x)-u_{2}(x)\right|=c\right\}\right|=0$; its complementary $Z^{c}=\Omega-Z$ is therefore the countable set, union of those sets such that $\left|\left\{x \in \Omega,\left|u_{1}(x)-u_{2}(x)\right|=c\right\}\right|>0$. Since for every $c$

$$
\nabla\left(u_{1}-u_{2}\right)=0 \quad \text { a.e. on }\left\{x \in \Omega,\left|u_{1}(x)-u_{2}(x)\right|=c\right\} \text {, }
$$

we obtain that

$$
\nabla\left(u_{1}-u_{2}\right)=0 \quad \text { a.e. on } Z^{c}
$$

Using Hölder inequality, we have

$$
\begin{aligned}
M= & \frac{1}{\beta} \int_{\left\{\left|u_{1}-u_{2}\right|>m\right\} \cap Z} b(x)\left(A_{0}+\left|\nabla u_{1}\right|+\left|\nabla u_{2}\right|\right)^{\frac{p-2}{\gamma}}\left|\nabla S_{m}\left(u_{1}-u_{2}\right)\right| \\
& \times\left(A_{0}+\left|\nabla u_{1}\right|+\left|\nabla u_{2}\right|\right)^{\sigma_{A_{0}}-\frac{p-2}{\gamma}}\left(B(x)+\left|\nabla u_{1}\right|+\left|\nabla u_{2}\right|\right)^{\sigma_{B}} \\
\leq & \frac{1}{\beta}\|b\|_{L^{r}\left(\left\{\left|u_{1}-u_{2}\right|>m\right\} \cap Z\right)}\left(\int_{\Omega}\left(A_{0}+\left|\nabla u_{1}\right|+\left|\nabla u_{2}\right|\right)^{p-2}\left|\nabla S_{m}\left(u_{1}-u_{2}\right)\right|^{\gamma}\right)^{\frac{1}{\gamma}} \\
& \times\left\|A_{0}+B+\left|\nabla u_{1}\right|+\left|\nabla u_{2}\right|\right\|_{L^{\rho}(\Omega)}^{\sigma_{B}+\sigma_{A_{0}}-\frac{p-2}{\gamma}}|\Omega|^{\frac{1}{t}},
\end{aligned}
$$

whenever we are able to find $\gamma, \rho$ and $t$ such that

$$
\begin{gathered}
\sigma_{A_{0}}-\frac{p-2}{\gamma} \geq 0 \\
\frac{1}{r}+\frac{1}{\gamma}+\frac{\sigma_{B}+\sigma_{A_{0}}-\frac{p-2}{\gamma}}{\rho}+\frac{1}{t}=1 \\
1 \leq \rho<\frac{N(p-1)}{N-1}=\rho_{*}, \\
1<t \leq+\infty
\end{gathered}
$$

where $\gamma$ satisfies $(2.32)$.

Since by hypothesis $(2.23)$ one has $\sigma_{A_{0}}>\frac{p-2}{\gamma_{*}}$, there exists some $\gamma_{0}, 0<\gamma_{0}<\gamma_{*}$, such that

$$
\sigma_{A_{0}}>\frac{p-2}{\gamma_{0}}
$$

On the other hand, setting

$$
F(\gamma, \rho)=\frac{1}{r}+\frac{1}{\gamma}\left(1-\frac{p-2}{\rho}\right)+\frac{\sigma_{A_{0}}+\sigma_{B}}{\rho}-1,
$$

we observe that hypothesis $(2.24)$ reads as $F\left(\gamma_{*}, \rho_{*}\right)<0$, so that we can choose $\gamma$ with $\gamma_{0}<\gamma<\gamma_{*}$ and close to $\gamma_{*}$ such that

$$
F\left(\gamma, \rho_{*}\right)<0 .
$$

We now choose $\rho$ with $1<\rho<\rho_{*}$ and close to $\rho_{*}$ such that

$$
F(\gamma, \rho)<0
$$

From now on we consider these values of $\gamma$ and $\rho$ as fixed; observe that (2.36) and (2.38) are satisfied. Setting $F(\gamma, \rho)=-\frac{1}{t}$ defines $t$ which satisfies (2.39) and (2.37). Therefore the above use of Hölder inequality is licit. 
We explicitly observe that for these values of $\gamma, \rho$ and $t, M$ is a finite number, which implies that the left-hand side of $(2.33)$ is finite.

Fourth step. From (2.33) and (2.35) we deduce that

$$
\begin{aligned}
\int_{\Omega}\left(A_{0}+\left|\nabla u_{1}\right|+\right. & \left.\left|\nabla u_{2}\right|\right)^{p-2}\left|\nabla S_{m}\left(u_{1}-u_{2}\right)\right|^{\gamma} \leq C_{0} \frac{1}{\beta^{\gamma}}\|b\|_{L^{r}\left(\left\{\left|u_{1}-u_{2}\right|>m\right\} \cap Z\right)}^{\gamma} \\
& \times \int_{\Omega}\left(A_{0}+\left|\nabla u_{1}\right|+\left|\nabla u_{2}\right|\right)^{p-2}\left|\nabla S_{m}\left(u_{1}-u_{2}\right)\right|^{\gamma}\left\|A_{0}+B+\left|\nabla u_{1}\right|+\left|\nabla u_{2}\right|\right\|_{L^{\rho}(\Omega)}^{\left(\sigma_{B}+\sigma_{A_{0}}\right) \gamma-(p-2)}|\Omega|^{\frac{\gamma}{t}}
\end{aligned}
$$

Since every term in this inequality is finite, we obtain that

$$
\left(1-C\|b\|_{L^{r}\left(\left\{\left|u_{1}-u_{2}\right|>m\right\} \cap Z\right)}^{\gamma}\right) \int_{\Omega}\left(A_{0}+\left|\nabla u_{1}\right|+\left|\nabla u_{2}\right|\right)^{p-2}\left|\nabla S_{m}\left(u_{1}-u_{2}\right)\right|^{\gamma} \leq 0,
$$

where $C$ is a constant which depends only on $N, s, p,\left\|\left|\nabla u_{1}\right|^{p-2}\right\|_{L^{s}(\Omega)},\left\|\left|\nabla u_{2}\right|^{p-2}\right\|_{L^{s}(\Omega)}, A_{0}, B, \sigma_{A_{0}}, \sigma_{B}, \beta, \gamma$, $\rho,|\Omega|$, and $t$, and will therefore be considered as fixed; in particular this constant does not depend on $m$.

Arguing as in the end of the fourth step of the proof of Theorem 1.7, we obtain that $u_{1}=u_{2}$, i.e. the desired result.

The proof of the comparison result stated in Remark 1.12 is very similar: it is indeed sufficient to use the test function $T_{k}\left(S_{m}\left(T_{2 n}\left(u_{1}\right)-T_{2 n}\left(u_{2}\right)\right)^{+}\right)$in place of $T_{k}\left(S_{m}\left(T_{2 n}\left(u_{1}\right)-\left(T_{2 n}\left(u_{2}\right)\right)\right)\right.$ in $(2.4)$ in the above proof.

\subsection{Proof of Theorem $\mathbf{1 . 1 6}$}

First step. Observe that $H\left(x, \nabla u_{1}\right)$ and $H\left(x, \nabla u_{2}\right)$ belong to $L^{1}(\Omega)$ thanks to (1.8), (1.9), (1.16), (1.41), (1.43), and (1.45). But, as said at the beginning of the proof of Theorem 1.7, the first step of its proof holds also for $p>2$, whenever $H\left(x, \nabla u_{1}\right)$ and $H\left(x, \nabla u_{2}\right)$ belong to $L^{1}(\Omega)$, therefore $(2.7)$ holds in the present case.

Second step. From hypothesis (1.39), we deduce that

$$
\frac{2(p-1)}{N-1} \leq p-2
$$

and, hence, from (1.43) that

$$
\sigma_{A_{0}}+\sigma_{B}<p-2
$$

This yields

$$
\left(A_{0}+\left|\nabla u_{1}\right|+\left|\nabla u_{2}\right|\right)^{p-2} \geq C\left(A_{0}, p\right)\left(A_{0}+\left|\nabla u_{1}\right|+\left|\nabla u_{2}\right|\right)^{\sigma_{A_{0}}+\sigma_{B}}
$$

where $C\left(A_{0}, p\right)$ is a constant depending only on $A_{0}$ and $p$.

On the other hand, using hypotheses (1.7), (1.9), and (1.40), we deduce from (2.7) that

$$
\begin{aligned}
\int_{\Omega}\left(A_{0}\right. & \left.+\left|\nabla u_{1}\right|+\left|\nabla u_{2}\right|\right)^{\sigma_{A_{0}}+\sigma_{B}}\left|\nabla T_{k}\left(S_{m}\left(u_{1}-u_{2}\right)\right)\right|^{2} \\
& \leq \frac{1}{\beta C\left(A_{0}, p\right)} \int_{\Omega}\left(A_{0}+\left|\nabla u_{1}\right|+\left|\nabla u_{2}\right|\right)^{p-2}\left|\nabla u_{1}-\nabla u_{2}\right|^{2} \chi_{\left\{\left|S_{m}\left(u_{1}-u_{2}\right)\right|<k\right\}} \leq M k,
\end{aligned}
$$

where $M$ is given by

$$
M=\frac{1}{\beta C\left(A_{0}, p\right)} \int_{\left\{\left|u_{1}-u_{2}\right|>m\right\}} b(x)\left(A_{0}+\left|\nabla u_{1}\right|+\left|\nabla u_{2}\right|\right)^{\sigma_{A_{0}}}\left(B(x)+\left|\nabla u_{1}\right|+\left|\nabla u_{2}\right|\right)^{\sigma_{B}}\left|\nabla u_{1}-\nabla u_{2}\right| .
$$


We now apply Lemma 4.2 with $v=\left(A_{0}+\left|\nabla u_{1}\right|+\left|\nabla u_{2}\right|\right)^{\sigma_{A_{0}}+\sigma_{B}}$. Observe that $v \geq A_{0}>0$ a.e. in $\Omega$ and that $v \in L^{s}(\Omega)$ with $s<\frac{N(p-1)}{(N-1)\left(\sigma_{A_{0}}+\sigma_{B}\right)}$; moreover $\frac{N(p-1)}{(N-1)\left(\sigma_{A_{0}}+\sigma_{B}\right)}>\frac{N}{2}$ since $\sigma_{A_{0}}+\sigma_{B}<\frac{2(p-1)}{N-1}$ when (1.43) is satisfied. Hence we can apply Lemma 4.2 with $s$ and $\gamma$ such that

$$
\left\{\begin{array}{l}
\frac{N}{2}<s<\frac{N(p-1)}{(N-1)\left(\sigma_{A_{0}}+\sigma_{B}\right)} \\
1<\gamma<\frac{2 N(s-1)}{N(2 s-1)-2 s}
\end{array}\right.
$$

A simple calculation shows that the second line of (2.42) is equivalent to

$$
1<\gamma<\frac{2(p-1) N-2(N-1)\left(\sigma_{A_{0}}+\sigma_{B}\right)}{(N-1)\left[2(p-1)-\left(\sigma_{A_{0}}+\sigma_{B}\right)\right]}=\gamma_{* *}
$$

We deduce from (2.40) and Lemma 4.2 that

$$
\int_{\Omega}\left(A_{0}+\left|\nabla u_{1}\right|+\left|\nabla u_{2}\right|\right)^{\sigma_{A_{0}}+\sigma_{B}}\left|\nabla S_{m}\left(u_{1}-u_{2}\right)\right|^{\gamma} \leq C_{0} M^{\gamma},
$$

where $C_{0}$ is a constant which only depends on $N, s, p, \sigma_{A_{0}}, \sigma_{B}, \gamma, A_{0},\left\|\left|\nabla u_{1}\right|^{p-2}\right\|_{L^{s}(\Omega)}$ and $\left\|\left|\nabla u_{2}\right|^{p-2}\right\|_{L^{s}(\Omega)}$.

Third step. In this step, we estimate $M$.

As in the proofs of Theorems 1.7 and 1.11, we introduce a set $Z$ in the following way: since $|\Omega|$ is finite, the set of the constants such that $\left|\left\{x \in \Omega,\left|u_{1}(x)-u_{2}(x)\right|=c\right\}\right|>0$ is at most countable. Let $Z \subset \Omega$ be the union of those sets such that $\left|\left\{x \in \Omega,\left|u_{1}(x)-u_{2}(x)\right|=c\right\}\right|=0$; its complementary $Z^{c}=\Omega-Z$ is therefore the countable set, union of those sets such that $\left|\left\{x \in \Omega,\left|u_{1}(x)-u_{2}(x)\right|=c\right\}\right|>0$. Since for every $c$

$$
\nabla\left(u_{1}-u_{2}\right)=0 \quad \text { a.e. on }\left\{x \in \Omega,\left|u_{1}(x)-u_{2}(x)\right|=c\right\} \text {, }
$$

we obtain that

Using Hölder inequality, we have

$$
\nabla\left(u_{1}-u_{2}\right)=0 \quad \text { a.e. on } Z^{c}
$$

$$
\begin{aligned}
& M=\frac{1}{\beta C\left(A_{0}, p\right)} \int_{\left\{\left|u_{1}-u_{2}\right|>m\right\} \cap Z} b(x)\left(A_{0}+\left|\nabla u_{1}\right|+\left|\nabla u_{2}\right|\right)^{\frac{\sigma_{A_{0}}+\sigma_{B}}{\gamma}}\left|\nabla S_{m}\left(u_{1}-u_{2}\right)\right| \\
& \times\left(A_{0}+\left|\nabla u_{1}\right|+\left|\nabla u_{2}\right|\right)^{\sigma_{A_{0}}-\frac{\sigma_{A_{0}}+\sigma_{B}}{\gamma}}\left(B(x)+\left|\nabla u_{1}\right|+\left|\nabla u_{2}\right|\right)^{\sigma_{B}} \\
& \leq \frac{\|b\|_{L^{r}\left(\left\{\left|u_{1}-u_{2}\right|>m\right\} \cap Z\right)}}{\beta C\left(A_{0}, p\right)}\left(\int_{\Omega}\left(A_{0}+\left|\nabla u_{1}\right|+\left|\nabla u_{2}\right|\right)^{\sigma_{A_{0}}+\sigma_{B}}\left|\nabla S_{m}\left(u_{1}-u_{2}\right)\right|^{\gamma}\right)^{\frac{1}{\gamma}} \\
& \times\left\|A_{0}+B+\left|\nabla u_{1}\right|+\left|\nabla u_{2}\right|\right\|_{L^{\rho}(\Omega)}^{\left(\sigma_{B}+\sigma_{A_{0}}\right)\left(1-\frac{1}{\gamma}\right)}|\Omega|^{\frac{1}{t}},
\end{aligned}
$$

whenever we are able to find $\gamma, \rho$ and $t$ such that

$$
\begin{gathered}
\sigma_{A_{0}}\left(1-\frac{1}{\gamma}\right) \geq \frac{\sigma_{B}}{\gamma}, \\
\frac{1}{r}+\frac{1}{\gamma}+\frac{\left(\sigma_{B}+\sigma_{A_{0}}\right)\left(1-\frac{1}{\gamma}\right)}{\rho}+\frac{1}{t}=1,
\end{gathered}
$$




$$
\begin{gathered}
1 \leq \rho<(p-1) \frac{N}{N-1}=\rho_{*}, \\
1<t \leq+\infty,
\end{gathered}
$$

where $\gamma$ satisfies (2.43). Since by (1.44), one has $\sigma_{A_{0}}\left(1-\frac{1}{\gamma_{* *}}\right)>\frac{\sigma_{B}}{\gamma_{* *}}$, there exists a $\gamma_{1}, 0<\gamma_{1}<\gamma_{* *}$ such that

$$
\sigma_{A_{0}}\left(1-\frac{1}{\gamma_{1}}\right)>\frac{\sigma_{B}}{\gamma_{1}}
$$

On the other hand, setting

$$
F(\gamma, \rho)=\frac{1}{r}+\frac{1}{\gamma}+\frac{\left(\sigma_{B}+\sigma_{A_{0}}\right)\left(1-\frac{1}{\gamma}\right)}{\rho}-1
$$

we observe that condition (1.43) reads as $F\left(\gamma_{* *}, \rho_{*}\right)<0$. Therefore, as in the third step of the proof of Theorem 1.11, we can choose the values of $\rho, \gamma$ and $t$ such that (2.47)-(2.49) and (2.50) holds true.

We explicitly observe that for these values of $\gamma, \rho$ and $t, M$ is a finite number, which implies that the left-hand side of (2.44) is finite.

Fourth step. From (2.44) and (2.46) we deduce that

$$
\begin{aligned}
\int_{\Omega}\left(A_{0}+\left|\nabla u_{1}\right|+\left|\nabla u_{2}\right|\right)^{\sigma_{B}+\sigma_{A_{0}}\left|\nabla S_{m}\left(u_{1}-u_{2}\right)\right|^{\gamma} \leq} & \frac{C_{0}\|b\|_{L^{r}\left(\left\{\left|u_{1}-u_{2}\right|>m\right\} \cap Z\right)}^{\gamma}}{\left(\beta C\left(A_{0}, p\right)\right)^{\gamma}} \\
& \times \int_{\Omega}\left(A_{0}+\left|\nabla u_{1}\right|+\left|\nabla u_{2}\right|\right)^{\sigma_{B}+\sigma_{A_{0}}}\left|\nabla S_{m}\left(u_{1}-u_{2}\right)\right|^{\gamma} \\
& \times\left\|A_{0}+B+\left|\nabla u_{1}\right|+\left|\nabla u_{2}\right|\right\|_{L^{\rho}(\Omega)}^{\left(\sigma_{B}+\sigma_{A_{0}}\right)(\gamma-1)}|\Omega|^{\frac{\gamma}{t}}
\end{aligned}
$$

Since every term in this inequality is finite, we obtain that

$$
\left(1-C\|b\|_{L^{r}\left(\left\{\left|u_{1}-u_{2}\right|>m\right\} \cap Z\right)}^{\gamma}\right) \int_{\Omega}\left(A_{0}+\left|\nabla u_{1}\right|+\left|\nabla u_{2}\right|\right)^{\sigma_{B}+\sigma_{A_{0}}}\left|\nabla S_{m}\left(u_{1}-u_{2}\right)\right|^{\gamma} \leq 0,
$$

where $C$ is a constant which depends only on $N, s, p,\left\|\left|\nabla u_{1}\right|^{p-2}\right\|_{L^{s}(\Omega)},\left\|\left|\nabla u_{2}\right|^{p-2}\right\|_{L^{s}(\Omega)}, A_{0}, B, \sigma_{A_{0}}, \sigma_{B}, \beta, \gamma$, $\rho,|\Omega|$ and $t$, and will therefore be considered as fixed; in particular this constant does not depend on $m$.

Arguing as in the end of the fourth step of the proof Theorem 1.7, we obtain that $u_{1}=u_{2}$, i.e. the desired result.

The proof of the comparison result stated in Remark 1.17 is very similar: it is indeed sufficient to use the test function $T_{k}\left(S_{m}\left(T_{2 n}\left(u_{1}\right)-T_{2 n}\left(u_{2}\right)\right)^{+}\right)$in place of $T_{k}\left(S_{m}\left(T_{2 n}\left(u_{1}\right)-\left(T_{2 n}\left(u_{2}\right)\right)\right)\right.$ in (2.4) in the above proof.

\section{Appendix 1: A property of the Difference of two RENORMALIZEd SOlutions}

In this section we consider renormalized solutions of equation (1.4) in the case where

$$
H(x, \xi)=0, \quad G(x, s)=0,
$$


that is renormalized solutions $u_{1}$ and $u_{2}$, in the sense of Definition 1.4, of the problems

$$
\begin{aligned}
& \begin{cases}-\operatorname{div}\left(a\left(x, \nabla u_{1}\right)\right)=\mu_{1} & \text { in } \Omega, \\
u_{1}=0 & \text { on } \partial \Omega,\end{cases} \\
& \begin{cases}-\operatorname{div}\left(a\left(x, \nabla u_{2}\right)\right)=\mu_{2} & \text { in } \Omega, \\
u_{2}=0 & \text { on } \partial \Omega,\end{cases}
\end{aligned}
$$

where $\mu_{1}=f_{1}-\operatorname{div}\left(g_{1}\right)$ and $\mu_{2}=f_{2}-\operatorname{div}\left(g_{2}\right)$, for given $f_{1}, f_{2}$ in $L^{1}(\Omega)$ and $g_{1}, g_{2}$ in $\left(L^{p^{\prime}}(\Omega)\right)^{N}$.

The following theorem is proved in [8] (Theorem 9.1) in the more general case where $\mu_{1}$ and $\mu_{2}$ are Radon measures with bounded total variation.

Theorem 3.1. Assume that a satisfies (1.5)-(1.11) and that $u_{1}$ and $u_{2}$ are renormalized solutions of (3.1) and (3.2). Then for every $k>0$, we have

$$
\left(a\left(x, \nabla u_{1}\right)-\left(a\left(x, \nabla u_{2}\right)\right) \cdot\left(\nabla u_{1}-\nabla u_{2}\right) \chi_{\left\{\left|u_{1}-u_{2}\right|<k\right\}} \in L^{1}(\Omega),\right.
$$

and

$$
\int_{\left\{\left|u_{1}-u_{2}\right|<k\right\}}\left(a\left(x, \nabla u_{1}\right)-\left(a\left(x, \nabla u_{2}\right)\right) \cdot\left(\nabla u_{1}-\nabla u_{2}\right) \leq\left[\left|\mu_{1}\right|(\Omega)+\left|\mu_{2}\right|(\Omega)\right)\right] k .
$$

In the case where $p \geq 2$ and where the strong monotonicity condition

$$
\left(a\left(x, \xi_{1}\right)-\left(a\left(x, \xi_{2}\right)\right) \cdot\left(\xi_{1}-\xi_{2}\right) \geq \alpha\left|\xi_{1}-\xi_{2}\right|^{p}, \alpha>0\right.
$$

holds, estimate (3.3) implies that $T_{k}\left(u_{1}-u_{2}\right) \in W_{0}^{1, p}(\Omega)$ for every $k>0$ and that

$$
\alpha \int_{\Omega}\left|\nabla T_{k}\left(u_{1}-u_{2}\right)\right|^{p} \leq\left[\left|\mu_{1}\right|(\Omega)+\left|\mu_{2}\right|(\Omega)\right] k
$$

(see [8], Theorem 9.2, where it is assumed, more in general, that $\mu_{1}$ and $\mu_{2}$ are Radon measures with bounded total variation).

The goal of the present section is to give an extension of the previous result to the case $p<2$. We assume here that

$$
\frac{3 N-2}{2 N-1}<p<2
$$

(observe that the case where $p$ is close to 1 is not covered).

Theorem 3.2. Assume that the hypotheses of Theorem 3.1 are satisfied, that $p$ satisfies (3.7), and that a( $x, \xi)$ satisfies the strong monotonicity condition (1.7).

Then for every $k>0$ we have

$$
T_{k}\left(u_{1}-u_{2}\right) \in W_{0}^{1, s}(\Omega) \quad \text { with } \quad s<\frac{2 N(p-1)}{N-(2-p)}
$$

and

$$
\alpha^{\frac{(2-p) s}{2(p-1)}} \beta^{\frac{s}{2}} \int_{\Omega}\left|\nabla T_{k}\left(u_{1}-u_{2}\right)\right|^{s} \leq C(N, p, s,|\Omega|)\left[\alpha\left\|A^{p-1}\right\|_{L^{N^{\prime}, \infty}(\Omega)}+\left|\mu_{1}\right|(\Omega)+\left|\mu_{2}\right|(\Omega)\right]^{\frac{s(2-p)}{2(p-1)}} k^{\frac{s}{2}},
$$

where $C(N, p, s,|\Omega|)$ is a constant depending only on $N, p, s$ and $|\Omega|$. 
Remark 3.3. Note that $\frac{2 N(p-1)}{N-(2-p)}>1$ when $p>\frac{3 N-2}{2 N-1}$, as we assumed in (3.7). On the other hand, the constant $C(N, p, s,|\Omega|)$ which appears in (3.9) tends to infinity when $s$ tends to $\frac{2 N(p-1)}{N-(2-p)}$. Note also that one could actually prove, by a proof similar to the proof below, that under the assumptions of Theorem 3.2, $\left|\nabla T_{k}\left(u_{1}-u_{2}\right)\right|$ belongs to the Lorentz space $L^{\hat{s}, 2}(\Omega)$, with $\hat{s}=\frac{2 N(p-1)}{N-(2-p)}$ and

$$
\left\|\left|\nabla T_{k}\left(u_{1}-u_{2}\right)\right|\right\|_{L^{\hat{s}, 2}(\Omega)}^{\hat{s}} \leq C(N, p, s,|\Omega|)\left[\alpha\left\|A^{p-1}\right\|_{L^{N^{\prime}, \infty}(\Omega)}+\left|\mu_{1}\right|(\Omega)+\left|\mu_{2}\right|(\Omega)\right]^{\frac{\hat{s}}{2}+\frac{(2-p) \hat{s}}{p-1}} k^{\frac{\hat{s}}{2}} .
$$

Proof of Theorem 3.2. The proof is based on the use of Hölder inequality and of the strong monotonicity assumption (1.7). Using Hölder inequality ${ }^{6}$ with the exponent $\frac{2}{s}, s<\frac{2 N(p-1)}{N-(2-p)}$ (note that $\frac{2}{s}>1$ ) we have

$$
\begin{aligned}
\int_{\left\{\left|u_{1}-u_{2}\right|<k\right\}}\left|\nabla u_{1}-\nabla u_{2}\right|^{s}= & \int_{\left\{\left|u_{1}-u_{2}\right|<k\right\}} \frac{\left|\nabla u_{1}-\nabla u_{2}\right|^{s}}{\left(A(x)+\left|\nabla u_{1}\right|+\left|\nabla u_{2}\right|\right)^{\frac{s(2-p)}{2}}}\left(A(x)+\left|\nabla u_{1}\right|+\left|\nabla u_{2}\right|\right)^{\frac{s(2-p)}{2}} \\
\leq & \left(\int_{\left\{\left|u_{1}-u_{2}\right|<k\right\}} \frac{\left|\nabla u_{1}-\nabla u_{2}\right|^{2}}{\left(A(x)+\left|\nabla u_{1}\right|+\left|\nabla u_{2}\right|\right)^{2-p}}\right)^{\frac{s}{2}} \\
& \times\left(\int_{\Omega}\left(A(x)+\left|\nabla u_{1}\right|+\left|\nabla u_{2}\right|\right)^{\frac{s(2-p)}{2-s}}\right)^{\frac{2-s}{2}} .
\end{aligned}
$$

By the strong monotonicity assumption (1.7) and Theorem 3.1, we have

$$
\int_{\left\{\left|u_{1}-u_{2}\right|<k\right\}} \frac{\left|\nabla u_{1}-\nabla u_{2}\right|^{2}}{\left(A(x)+\left|\nabla u_{1}\right|+\left|\nabla u_{2}\right|\right)^{2-p}} \leq \frac{1}{\beta}\left[\left|\mu_{1}\right|(\Omega)+\left|\mu_{2}\right|(\Omega)\right] k .
$$

On the other hand, when $s<\frac{2 N(p-1)}{N-(2-p)}$, one has

$$
\left(\int_{\Omega}\left(A+\left|\nabla u_{1}\right|+\left|\nabla u_{2}\right|\right)^{\frac{s(2-p)}{(2-s)}}\right)^{\frac{(2-s)}{s(2-p)}} \leq C(s,|\Omega|)\left\|\left(A+\left|\nabla u_{1}\right|+\left|\nabla u_{2}\right|\right)^{p-1}\right\|_{L^{N^{\prime}, \infty}(\Omega)}^{\frac{1}{p-1}}
$$

Indeed, if $s \geq 2(p-1)$, then $\frac{s(2-p)}{(2-s)(p-1)} \geq 1$; moreover when $s<\frac{2 N(p-1)}{N-(2-p)}$, one has $N^{\prime} \geq \frac{s(2-p)}{(2-s)(p-1)}$. Therefore since $\Omega$ is bounded,

$$
L^{N^{\prime}, \infty}(\Omega) \subset L^{\frac{s(2-p)}{(2-s)(p-1)}}(\Omega)
$$

and inequality (3.13) holds true.

If $s<2(p-1)$, then $\frac{s(2-p)}{(2-s)(p-1)}<1$; using Hölder inequality with the exponent $\frac{(2-s)(p-1)}{s(2-p)}>1$ we get again (3.13).

\footnotetext{
${ }^{6}$ Actually one has to exclude the set $\left\{x \in \Omega: \nabla u_{1}(x)=\nabla u_{2}(x)=0\right\}$ in the integrals which are considered in this computation.
} 
From (3.13) we have

$$
\begin{aligned}
& \left(\int_{\Omega}\left(A+\left|\nabla u_{1}\right|+\left|\nabla u_{2}\right|\right)^{\frac{s(2-p)}{(2-s)}}\right)^{\frac{(2-s)}{s(2-p)}} \\
& \quad \leq C(s,|\Omega|, p)\left(\left\|A^{p-1}\right\|_{L^{N^{\prime}, \infty}(\Omega)}+\left\|\left|\nabla u_{1}\right|^{p-1}\right\|_{L^{N^{\prime}, \infty}(\Omega)}+\left\|\left|\nabla u_{2}\right|^{p-1}\right\|_{L^{N^{\prime}, \infty}(\Omega)}\right)^{\frac{1}{p-1}}
\end{aligned}
$$

From the estimate

$$
\int_{\Omega}\left|\nabla T_{k}\left(u_{1}\right)\right|^{p} \leq \frac{\left|\mu_{1}\right|(\Omega)}{\alpha} k
$$

which holds for every renormalized solution of (3.1) (this estimate is nothing but (3.4) in the case where $u_{2}=\mu_{2}=0$, since $a(x, 0)=0$ in view of $\left.(1.5)\right)$ we deduce, using Lemma 4.3 of Appendix 2 below with $L=0$ that

$$
\left\|\left|\nabla u_{1}\right|^{p-1}\right\|_{L^{N^{\prime}, \infty}(\Omega)} \leq C(N, p) \frac{\left|\mu_{1}\right|(\Omega)}{\alpha} .
$$

A similar estimate holds true for $u_{2}$ and therefore we deduce from (3.14) that

$$
\left(\int_{\Omega}\left(A+\left|\nabla u_{1}\right|+\left|\nabla u_{2}\right|\right)^{\frac{s(2-p)}{(2-s)}}\right)^{\frac{(2-s)}{s(2-p)}} \leq \frac{C(N, s,|\Omega|, p)}{\alpha^{\frac{1}{p-1}}}\left(\alpha\left\|A^{p-1}\right\|_{L^{N^{\prime}, \infty}(\Omega)}+\left|\mu_{1}\right|(\Omega)+\left|\mu_{2}\right|(\Omega)\right)^{\frac{1}{p-1}}
$$

Combining (3.11), (3.12) and (3.15) we have

$$
\alpha^{\frac{(2-p) s}{2(p-1)}} \beta^{\frac{s}{2}} \int_{\left\{\left|u_{1}-u_{2}\right|<k\right\}}\left|\nabla u_{1}-\nabla u_{2}\right|^{s} \leq C(N, p, s,|\Omega|)\left(\alpha\left\|A^{p-1}\right\|_{L^{N^{\prime}, \infty}(\Omega)}+\left|\mu_{1}\right|(\Omega)+\left|\mu_{2}\right|(\Omega)\right)^{\frac{s(2-p)}{2(p-1)}} k^{\frac{s}{2}} .
$$

Estimate (3.16) is very similar to the result (3.9) of Theorem 3.2, but there is still a difficulty. Indeed, since $u_{1}$ and $u_{2}$ are renormalized solutions of (3.1) and (3.2), their gradients $\nabla u_{1}$ and $\nabla u_{2}$ are defined by (1.3) and this does not allow one to write $\nabla\left(u_{1}-u_{2}\right)=\nabla u_{1}-\nabla u_{2}$ (actually we do not know yet that the $\nabla\left(u_{1}-u_{2}\right)$ exists). To solve this difficulty, we proceed like in [8], Proof of Theorem 9.2. It is easy to see that for $n>k$

$$
\int_{\Omega}\left|\nabla T_{k}\left(T_{n}\left(u_{1}\right)-T_{n}\left(u_{2}\right)\right)\right|^{s} \leq \int_{\left\{\left|u_{1}-u_{2}\right|<k\right\}}\left|\nabla u_{1}-\nabla u_{2}\right|^{s}+\int_{\left\{n-k<\left|u_{1}\right|<n\right\}}\left|\nabla u_{1}\right|^{s}+\int_{\left\{n-k<\left|u_{2}\right|<n\right\}}\left|\nabla u_{2}\right|^{s} .
$$

For each $k$ fixed, the right-hand side of (3.17) is bounded independently of $n$ in view of (3.16) and of the following result (see $(5.10)$ of $[8])^{7}$ :

$$
\begin{aligned}
& \frac{1}{k} \int_{n<\left|u_{1}\right|<n+k}\left|\nabla u_{1}\right|^{s} \leq c, \\
& \frac{1}{k} \int_{n<\left|u_{2}\right|<n+k}\left|\nabla u_{2}\right|^{s} \leq c .
\end{aligned}
$$

Therefore the function $T_{k}\left(T_{n}\left(u_{1}\right)-T_{n}\left(u_{2}\right)\right)$ is bounded in $W_{0}^{1, s}(\Omega)$ uniformly with respect to $n$. Since this function converges to $T_{k}\left(u_{1}-u_{2}\right)$ almost everywhere in $\Omega$ as $n$ tends to infinity, we conclude that $T_{k}\left(u_{1}-u_{2}\right)$

\footnotetext{
${ }^{7}$ This result is formally obtained by using $S_{n-k, n}\left(u_{1}\right)$ as test function in (3.1) and using the coerciveness. This proof becomes correct, thanks to (1.17), if one uses the test function $S_{n-k, n}\left(u_{1}\right) h_{j}\left(u_{1}\right)$, with $h_{j}$ defined by (1.22) and let $j$ tends to infinity
} 
belong to $W_{0}^{1, s}(\Omega)$ for every $k>0$. Using Lemma 2.12 of [8], we have

$$
\left(\nabla u_{1}-\nabla u_{2}\right) \chi_{\left\{\left|u_{1}-u_{2}\right|<k\right\}}=\nabla\left(u_{1}-u_{2}\right) \chi_{\left\{\left|u_{1}-u_{2}\right|<k\right\}}=\nabla T_{k}\left(u_{1}-u_{2}\right),
$$

which allows us to deduce (3.9) from (3.16).

\section{Appendix 2: Some extensions of a Result of [1]}

In this section we extend in two different ways a result of Bénilan et al. ([1], Lemmas 4.1 and 4.2) and we recall an extension of it (Lemma 4.3) which we have proved in [3] .

Lemma 4.1. Assume that $\Omega$ is an open subset of $\mathbb{R}^{N}$ with finite measure and that $1 \leq s<N$. Let $u$ be a measurable function satisfying $T_{k}(u) \in W_{0}^{1, s}(\Omega)$, for every positive $k$, and such that

$$
\int_{\Omega}\left|\nabla T_{k}(u)\right|^{s} \leq M k^{\theta} \quad \forall k>0
$$

where $M$ is a given constant and $0<\theta<s$. Then $|u|^{s-\theta}$ belongs to $L^{\frac{N}{N-s}, \infty}(\Omega),|\nabla u|^{s-\theta}$ belongs to $L^{\frac{N}{N-\theta}, \infty}(\Omega)$ and

$$
\begin{gathered}
\left\||u|^{s-\theta}\right\|_{L^{\frac{N}{N-s}, \infty}(\Omega)} \leq C(N, s) M, \\
\left\||\nabla u|^{s-\theta}\right\|_{L^{\frac{N}{N-\theta}, \infty}(\Omega)} \leq C(N, s) M,
\end{gathered}
$$

where $C(N, s)$ is a constant depending only on $N$ and $s$.

Proof of Lemma 4.1.

Proof of (4.2). From Sobolev inequality, there exists a constant $S_{N, p}$ which depends only on $N$ and $p$, such that for every open set $\Omega \subset \mathbb{R}^{N}$ and $v \in W_{0}^{1, p}(\Omega)$, one has

$$
\|v\|_{L^{p^{*}(\Omega)}}^{p^{*}} \leq S_{N, p}\|\nabla v\|_{L^{p}(\Omega)}^{p^{*}} .
$$

Therefore we have, for every $k>0$

$$
k^{s^{*}} \operatorname{meas}\{x \in \Omega:|u|>k\} \leq \int_{\Omega}\left|T_{k}(u)\right|^{s^{*}} \leq S_{N, s}\left\|\left|\nabla T_{k}(u)\right|\right\|_{L^{s}(\Omega)}^{s^{*}} \leq S_{N, s}\left(M k^{\theta}\right)^{\frac{s^{*}}{s}},
$$

or equivalently, for every $h>0$

$$
h^{\frac{s^{*}}{s-\theta}} \operatorname{meas}\left\{x \in \Omega:|u|^{s-\theta}>h\right\} \leq S_{N, s}\left(M h^{\frac{\theta}{s-\theta}}\right)^{\frac{s^{*}}{s}} .
$$

We deduce that

$$
\operatorname{meas}\left\{x \in \Omega:|u|^{s-\theta}>h\right\} \leq S_{N, s} M^{\frac{s^{*}}{s}} h^{\left(\frac{\theta}{s}-1\right) \frac{s^{*}}{s-\theta}},
$$

and

$$
h\left(\operatorname{meas}\left\{x \in \Omega:|u|^{s-\theta}>h\right\}\right)^{\frac{N-s}{N}} \leq S_{N, s}^{\frac{N-s}{N}} M,
$$

for every $h>0$. This yields (4.2). 
Proof of (4.3). From (4.1) we deduce that for every $\lambda>0$ and every $k>0$

$$
\lambda^{s} \operatorname{meas}\{x \in \Omega:|\nabla u|>\lambda \text { and }|u| \leq k\} \leq \int_{\{|u| \leq k\}}|\nabla u|^{s}=\int_{\{|u| \leq k\}}\left|\nabla T_{k}(u)\right|^{s} \leq M k^{\theta},
$$

i.e. for every $\mu>0$ and every $k>0$

$$
\mu^{\frac{s}{s-\theta}} \operatorname{meas}\left\{x \in \Omega:|\nabla u|^{s-\theta}>\mu \text { and }|u| \leq k\right\} \leq M k^{\theta} .
$$

From (4.6) and (4.5), we obtain that for every $\lambda>0$ and every $k>0$

$$
\begin{aligned}
\operatorname{meas}\left\{x \in \Omega:|\nabla u|^{s-\theta}>\mu\right\} \leq & \operatorname{meas}\left\{x \in \Omega:|\nabla u|^{s-\theta}>\mu \text { and }|u| \leq k\right\} \\
& +\operatorname{meas}\left\{x \in \Omega:|\nabla u|^{s-\theta}>\mu \text { and }|u|>k\right\} \\
\leq & C(N, s)\left(\frac{M k^{\theta}}{\mu^{\frac{s}{s-\theta}}}+\frac{M^{\frac{s^{*}}{s}}}{k^{\left(1-\frac{\theta}{s}\right) s^{*}}}\right) .
\end{aligned}
$$

Choosing

$$
k=M^{\frac{1}{N-\theta}} \mu^{\frac{N-s}{(N-\theta)(s-\theta)}}
$$

(this value minimizes with respect to $k$ the right-hand side of $(4.7)$ ), inequality (4.7) yields

$$
\operatorname{meas}\left\{x \in \Omega:|\nabla u|^{s-\theta}>\mu\right\} \leq C(N, s) \frac{M^{\frac{N}{N-\theta}}}{\mu^{\frac{N}{N-\theta}}}
$$

that is

which proves (4.2).

$$
\mu\left(\operatorname{meas}\left\{x \in \Omega:|\nabla u|^{s-\theta}>\mu\right\}\right)^{\frac{N-\theta}{N}} \leq C(N, s) M
$$

Lemma 4.2. Assume that $\Omega$ is an open subset of $\mathbb{R}^{N}$ with finite measure. Let $u$ be a measurable function satisfying $T_{k}(u) \in H_{0}^{1}(\Omega)$, for every positive $k$, and such that

$$
\int_{\Omega} v(x)\left|\nabla T_{k}(u)\right|^{2} \leq M k, \quad \forall k>0
$$

where $M$ is a given constant, and $v$ is a function such that

$$
\begin{gathered}
v(x) \geq A_{0}>0, \quad \text { a.e. in } \Omega, \\
v \in L^{s}(\Omega), \quad \text { with } \quad s>N / 2>1 .
\end{gathered}
$$

Then it results

$$
\int_{\Omega} v(x)|\nabla u|^{\gamma} \leq C_{0} M^{\gamma}
$$


where

$$
1 \leq \gamma<\frac{2 N(s-1)}{2(N-1) s-N}
$$

and $C_{0}$ is a positive constant depending only on $A_{0}, N, s, \gamma$ and $\|v\|_{L^{s}(\Omega)}$.

Proof of Lemma 4.2. For every $\varepsilon>0$, we have:

$$
\int_{\Omega} v(x)|\nabla u|^{\gamma}=\int_{\{|u|<\varepsilon\}} v(x)|\nabla u|^{\gamma}+\sum_{n=0}^{\infty} \int_{\left\{2^{n} \varepsilon \leq|u|<2^{n+1} \varepsilon\right\}} v(x)|\nabla u|^{\gamma} .
$$

We begin by estimating the first integral in the right-hand side of (4.13). Using (4.8) and Hölder inequality we have

$$
\begin{aligned}
\int_{\{|u|<\varepsilon\}} v(x)|\nabla u|^{\gamma} & \leq\left(\int_{\{|u|<\varepsilon\}} v(x)|\nabla u|^{2}\right)^{\frac{\gamma}{2}}\left(\int_{\{|u|<\varepsilon\}} v(x)\right)^{1-\frac{\gamma}{2}} \leq\left(\int_{\Omega} v(x)\left|\nabla T_{\varepsilon}(u)\right|^{2}\right)^{\frac{\gamma}{2}}\|v\|_{L^{1}(\Omega)}^{1-\frac{\gamma}{2}} \\
& \leq\|v\|_{L^{1}(\Omega)}^{1-\frac{\gamma}{2}}(M \varepsilon)^{\frac{\gamma}{2}} .
\end{aligned}
$$

Now we estimate the integrals $\int_{\left\{2^{n} \varepsilon \leq|u|<2^{n+1} \varepsilon\right\}} v(x)|\nabla u|^{\gamma}$.

Setting $k=2^{n} \varepsilon$, Hölder inequality and (4.8) give

$$
\begin{aligned}
\int_{\{k \leq|u|<2 k\}} v(x)|\nabla u|^{\gamma} \leq & \left(\int_{\{k \leq|u|<2 k\}} v(x)|\nabla u|^{2}\right)^{\frac{\gamma}{2}}\left(\int_{\{k \leq|u|<2 k\}} v(x)\right)^{1-\frac{\gamma}{2}} \\
\leq & \left(\int_{\Omega} v(x)\left|\nabla T_{2 k}(u)\right|^{2}\right)^{\frac{\gamma}{2}}\left(\int_{\{k \leq|u|<2 k\}} v(x)^{s}\right)^{\left(1-\frac{\gamma}{2}\right) \frac{1}{s}} \\
& \times|\{k \leq|u|<2 k\}|^{\left(1-\frac{\gamma}{2}\right) \frac{1}{s^{\prime}}} \leq 2^{\frac{\gamma}{2}}(M k)^{\frac{\gamma}{2}}\|v\|_{s}^{1-\frac{\gamma}{2}}|\{k \leq|u|<2 k\}|^{\left(1-\frac{\gamma}{2}\right) \frac{1}{s^{\prime}}} .
\end{aligned}
$$

On the other hand, using Sobolev inequality, and hypotheses (4.8) and (4.9), we have for every $k>0$

$$
k^{2^{*}}|\{k \leq|u|<2 k\}| \leq \int_{\Omega}\left|T_{k}(u)\right|^{2^{*}} \leq S_{N}\left(\int_{\Omega}\left|\nabla T_{k}(u)\right|^{2}\right)^{\frac{2^{*}}{2}} \leq \frac{S_{N}}{A_{0}^{\frac{2^{*}}{2}}}(M k)^{\frac{2^{*}}{2}},
$$

where $S_{N}=S_{N, 2}$ is a positive constant depending only on $N$.

Therefore from (4.15) and (4.16) we have

$$
\int_{\{k \leq|u|<2 k\}} v(x)|\nabla u|^{\gamma} \leq\left(\frac{S_{N}}{A_{0}^{\frac{2^{*}}{2}}}\right)^{\left(1-\frac{\gamma}{2}\right) \frac{1}{s^{\prime}}}\|v\|_{s}^{1-\frac{\gamma}{2}} 2^{\frac{\gamma}{2}} M^{\alpha} k^{-\beta},
$$

where

$$
\beta=-\frac{\gamma}{2}+\left(1-\frac{\gamma}{2}\right) \frac{2^{*}}{2 s^{\prime}}, \quad \alpha=\frac{\gamma}{2}+\left(1-\frac{\gamma}{2}\right) \frac{2^{*}}{2 s^{\prime}} .
$$

Since $\gamma$ satisfies (4.12), we explicitely observe that $\beta>0$. 
Combining (4.13), (4.14), and (4.17), we get

$$
\begin{aligned}
\int_{\Omega} v(x)|\nabla u|^{\gamma} & \leq\|v\|_{L^{1}(\Omega)}^{1-\frac{\gamma}{2}} M^{\frac{\gamma}{2}} \varepsilon^{\frac{\gamma}{2}}+\sum_{n=0}^{\infty}\left(\frac{S_{N}}{A_{0}^{\frac{2^{*}}{2}}}\right)^{\left(1-\frac{\gamma}{2}\right) \frac{1}{s^{\prime}}}\|v\|_{s}^{1-\frac{\gamma}{2}} 2^{\frac{\gamma}{2}-n \beta} M^{\alpha} \varepsilon^{-\beta} \\
& \leq C\left[M^{\frac{\gamma}{2}} \varepsilon^{\frac{\gamma}{2}}+M^{\alpha} \varepsilon^{-\beta}\right]
\end{aligned}
$$

where $C$ is a constant depending only on $A_{0}, N, s, \gamma$ and $\|v\|_{L^{1}(\Omega)}$.

Choosing

$$
\varepsilon=M^{\frac{\alpha-\frac{\gamma}{2}}{\beta+\frac{1}{2}}}
$$

(this value minimizes with respect to $\varepsilon$ the right-hand side of (4.18)), inequality (4.18) gives (4.11).

Finally we recall the following lemma, that we proved in [3]:

Lemma 4.3. Assume that $\Omega$ is an open subset of $\mathbb{R}^{N}$ with finite measure and that $1<p<N$. Let $u$ be a measurable function satisfying $T_{k}(u) \in W_{0}^{1, p}(\Omega)$, for every positive $k$, and such that

$$
\int_{\Omega}\left|\nabla T_{k}(u)\right|^{p} \leq M k+L, \quad \forall k>0,
$$

where $M$ and $L$ are given constants. Then $|u|^{p-1}$ belongs to $L^{\frac{p^{*}}{p}, \infty}(\Omega),|\nabla u|^{p-1}$ belongs to $L^{N^{\prime}, \infty}(\Omega)$ and

$$
\begin{gathered}
\left\||u|^{p-1}\right\|_{L^{\frac{p^{*}}{p}, \infty}(\Omega)} \leq C(N, p)\left[M+|\Omega|^{\frac{1}{p^{*}}} L^{\frac{1}{p^{\prime}}}\right] \\
\left\||\nabla u|^{p-1}\right\|_{L^{N^{\prime}, \infty}(\Omega)} \leq C(N, p)\left[M+|\Omega|^{\frac{1}{N^{\prime}}-\frac{1}{p^{\prime}}} L^{\frac{1}{p^{\prime}}}\right],
\end{gathered}
$$

where $C(N, p)$ is a constant depending only on $N$ and $p$ and where $\frac{1}{p^{*}}=\frac{1}{p}-\frac{1}{N}$.

This work began during the visits made by the third author to the Università di Roma "La Sapienza" and to the Università di Napoli "Federico II". It was completed during the long term visit (September 2000 - July 2001) made by the second author to the Laboratoire d'Analyse Numérique de Université Pierre et Marie Curie (Paris VI) with the partial support of MURST. Both authors would like to thank these institutions for their hospitality.

\section{REFERENCES}

[1] P. Bénilan, L. Boccardo, T. Gallouët, R. Gariepy, M. Pierre and J.L. Vazquez, An $L^{1}$-theory of existence and uniqueness of solutions of nonlinear elliptic equations. Ann. Scuola Norm. Sup. Pisa Cl. Sci. 22 (1995) 241-273.

[2] M.F. Betta, A. Mercaldo, F. Murat and M.M. Porzio, Existence and uniqueness results for nonlinear elliptic problems with a lower order term and measure datum. C. R. Acad. Sci. Paris Sér. I Math. 332 (to appear).

[3] M.F. Betta, A. Mercaldo, F. Murat and M.M. Porzio, Existence of renormalized solutions to nonlinear elliptic equations with a lower order term and right-hand side measure. J. Math. Pures Appl. (to appear).

[4] M.F. Betta, A. Mercaldo, F. Murat and M.M. Porzio, Uniqueness results for nonlinear elliptic equations with a lower order term (to appear).

[5] L. Boccardo, T. Gallouët and L. Orsina, Existence and uniqueness of entropy solutions for nonlinear elliptic equations with measure data. Ann. Inst. H. Poincaré Anal. Non Linéaire 13 (1996) 539-551.

[6] G. Bottaro and M.E. Marina, Problema di Dirichlet per equazioni ellittiche di tipo variazionale su insiemi non limitati. Boll. Un. Mat. Ital. 8 (1973) 46-56.

[7] A. Dall'Aglio, Approximated solutions of equations with $L^{1}$ data. Application to the $H$-convergence of quasi-linear parabolic equations. Ann. Mat. Pura Appl. 170 (1996) 207-240. 
[8] G. Dal Maso, F. Murat, L. Orsina and A. Prignet, Renormalized solutions for elliptic equations with general measure data. Ann. Scuola Norm. Sup. Pisa Cl. Sci. 28 (1999) 741-808.

[9] G. Dolzmann, N. Hungerbühler and S. Müller, Uniqueness and maximal regularity for nonlinear elliptic systems of $n$-Laplace type with measure valued right-hand side. J. Reine Angew. Math. 520 (2000) 1-35.

[10] A. Fiorenza and C. Sbordone, Existence and uniqueness results for solutions of nonlinear equations with right-hand side in $L^{1}(\Omega)$. Studia Math. 127 (1998) 223-231.

[11] L. Greco, T. Iwaniec and C. Sbordone, Inverting the p-harmonic operator. Manuscripta Math. 92 (1997) 249-258.

[12] O. Guibé, Remarks on the uniqueness of comparable renormalized solutions of elliptic equations with measure data. Ann. Mat. Pura Appl. Ser. IV 180 (2002) 441-449.

[13] P.-L. Lions and F. Murat, Solutions renormalisées d'équations elliptiques non linéaires (to appear).

[14] F. Murat, Soluciones renormalizadas de EDP elipticas no lineales, Preprint 93023. Laboratoire d'Analyse Numérique de l'Université Paris VI (1993).

[15] F. Murat, Équations elliptiques non linéaires avec second membre $L^{1}$ ou mesure, in Actes du $26^{e}$ Congrès National d'Analyse Numérique. Les Karellis, France (1994) A12-A24.

[16] A. Prignet, Remarks on existence and uniqueness of solutions of elliptic problems with right-hand side measures. Rend. Mat. Appl. 15 (1995) 321-337.

[17] J. Serrin, Pathological solutions of elliptic differential equations. Ann. Scuola Norm. Sup. Pisa Cl. Sci. 18 (1964) 385-387.

[18] G. Stampacchia, Le problème de Dirichlet pour les équations elliptiques du second ordre à coefficients discontinus. Ann. Inst. Fourier (Grenoble) 15 (1965) 189-258. 